\title{
Trois mises en œuvre d'une transformation chimique pour introduire le thème des piles : des choix didactiques très contrastés
}

Three enactments of a chemical change to introduce the galvanic cells theme: some very different didactic choices

Isabelle Kermen et Philippe Colin

\section{OpenEdition Journals}

Édition électronique

URL : https://journals.openedition.org/educationdidactique/2772

DOI : $10.4000 /$ educationdidactique.2772

ISSN : 2111-4838

\section{Éditeur}

Presses universitaires de Rennes

\section{Édition imprimée}

Date de publication : 6 décembre 2017

Pagination : 187-212

ISBN : 978-2-7535-7318-5

ISSN : $1956-3485$

\section{Référence électronique}

Isabelle Kermen et Philippe Colin, « Trois mises en œuvre d'une transformation chimique pour introduire le thème des piles : des choix didactiques très contrastés ", Éducation et didactique [En ligne], 11-2 | 2017, mis en ligne le 06 décembre 2019, consulté le 22 juillet 2022. URL : http:// journals.openedition.org/educationdidactique/2772 ; DOI : https://doi.org/10.4000/ educationdidactique. 2772 


\title{
TROIS MISES EN GUVRE D'UNE TRANSFORMATION CHIMIQUE POUR INTRODUIRE LE THÈME DES PILES : DES CHOIX DIDACTIQUES TRÈS CONTRASTÉS
}

\author{
Isabelle Kermen \\ Universités d'Artois, laboratoire de didactique André Revuz, LDAR (EA 4434), universités d'Artois, \\ de Cergy-Pontoise, Paris Diderot, Paris Est Créteil, de Rouen \\ Philippe Colin \\ Universités d'Artois, laboratoire de didactique André Revuz, LDAR (EA 4434), universités d'Artois, \\ de Cergy-Pontoise, Paris Diderot, Paris Est Créteil, de Rouen
}

Lactivité de trois enseignantes de physique-chimie, lors d'une séance de TP sur les piles en terminale S, est analysée selon le cadre de la double approche didactique et ergonomique. L'étude centrée sur la réalisation de la première tâche, s'attache à montrer en quoi les choix du dispositif expérimental et du questionnement associé impactent le déroulement de chaque séance et en quoi les enseignantes diffèrent dans leur prise en compte du niveau expérimental, plus particulièrement des observations, et du modèle. Les différences constatées illustrent la marge de manœuvre qu'elles investissent, signe de l'influence de la composante personnelle de l'activité de chaque enseignante sur ses composantes cognitive et médiative, malgré une contrainte institutionnelle forte dans une classe d'examen.

Mots-clés : didactique de la chimie, modèle, prévision d'une transformation chimique, double approche didactique et ergonomique, activité enseignante.

Three enactments of a chemical change to introduce the galvanic cells theme: some very different didactic choices

The activity of three physics and chemistry teachers, conducting a session on galvanic cells in a 12th grade form, is analysed following the framework of the double didactic and ergonomic approach. The study focuses on the first task and attempts to show how the choices of the experimental device and of the associated questions impact the progress of each session and how the teachers take into account the experimental level, specifically the observations, and the model in a different way. The differences we observed illustrate what room for manoeuvre they have, which is a sign of the influence of the personal dimension of each teacher's activity on the cognitive and mediative dimensions of this activity, in spite of the strong institutional constraint of the final national exam.

Keywords: chemistry education, model, chemical change prediction, double didactic and ergonomic approach, teacher's activity. 
Isabelle Kermen \& Philippe Colin

\section{INTRODUCTION}

Cet article compare l'activité en classe de trois enseignantes à un même niveau d'enseignement (terminale scientifique en France) et lors d'une séance de travaux pratiques portant sur le même thème, les piles électrochimiques. Il s'inscrit dans un projet plus global visant à étudier l'activité enseignante en classe pour caractériser les pratiques d'enseignants de physique-chimie. Les études didactiques consacrées aux pratiques ordinaires d'enseignants de physique-chimie au lycée sont encore peu nombreuses (Cross, 2010; Tiberghien, 2012; Venturini, Calmettes, Amade-Escot, \& Terrisse, 2007). L'un des objectifs du projet consiste à mieux connaitre ces pratiques, pour chercher à identifier les raisons qui les sous-tendent et les contraintes qui pèsent sur elles. Le choix d'un même sujet est délibéré, il permet de repérer d'éventuelles régularités et des variabilités interindividuelles (Robert \& Vivier, 2013). Notre étude s'appuie sur le cadre de la double approche didactique et ergonomique développé par Robert et Rogalski (2002).

Notre article s'articule en sept parties. Dans la première nous précisons le cadre de la double approche, dans la deuxième le contexte de l'étude qui comporte une analyse du savoir en jeu, ensuite nous exposons notre problématique avec nos questions de recherche, que nous faisons suivre par la méthodologie de recueil et d'analyse des données. Nos résultats font l'objet des cinquième et sixième parties dans lesquelles nous présentons l'analyse a priori des fiches TP, puis l'analyse des déroulements des séances. Nous terminons par une synthèse et discussion des résultats pour les mettre en perspective.

\section{FONDEMENTS THÉORIQUES ET MÉTHODOLOGIQUES}

\section{La double approche didactique et ergonomique}

Le cadre de la double approche s'inscrit « dans la lignée des théories de l'activité » (Rogalski, 2003 référant à Leontiev, 1975). D'un point de vue de psychologie ergonomique, l'activité est la réponse que le sujet met en ouvre pour accomplir une tâche (Rogalski, 2003), elle comprend les actes extériorisés mais aussi ce qu'on ne voit pas, les hypothèses, les décisions (Rogalski, 2003). Ce cadre propose l'étude des pratiques enseignantes au travers de l'examen des activités possibles des élèves dans les séances observées pour reconstituer les choix didactiques et pédagogiques de l'enseignant (Robert, 2006) dans une visée compréhensive (Goigoux, 2007). Les pratiques d'un enseignant incluent tout ce qu'il dit, fait, pense ou non (Robert, 2008a) pour accomplir sa tâche: provoquer l'apprentissage d'un contenu donné par des élèves donnés et concevoir l'environnement d'apprentissage nécessaire à cela. Ce cadre a été proposé pour décrire et expliquer les régularités et la variabilité des pratiques des enseignants de mathématiques, dans la mesure où une analyse didactique (itinéraire cognitif et accompagnement proposé aux élèves par les enseignants) ne suffisait pas pour comprendre les choix faits par les enseignants (Robert \& Rogalski, 2002). Il était nécessaire de prendre en compte des facteurs extérieurs à la classe tels que le programme, les habitudes professionnelles, les représentations de l'enseignant lui-même (Robert \& Rogalski, 2002). Analyser les pratiques revient alors à analyser le travail d'un enseignant en tant que personne singulière en tenant compte des contraintes auxquelles il fait face (aspect ergonomique) et des particularités des situations d'enseignement-apprentissage (aspect didactique)

L'activité d'un enseignant désigne une partie délimitée de ses pratiques dans le temps et dans l'espace (Robert, 2012) ; l'activité en classe constitue ce qu'il développe durant une séance de classe pour parvenir au but qu'il s'est fixé, enseigner tels contenus à tels élèves. L'activité est distincte de, mais orientée par, la tâche qui est le but à atteindre dans des conditions données (Leplat \& Hoc, 1983). Les tâches de l'enseignant sont définies et prescrites par l'institution dans laquelle il travaille, les tâches effectives sont inférées de l'activité de l'enseignant et résultent de la représentation qu'il se fait des tâches à accomplir (Rogalski, 2003). Des enseignants différents ont une activité différente parce qu'ils ne lisent pas nécessairement les prescriptions de la même manière et s'en font donc une représentation singulière. De même, les élèves ont une représentation des tâches qui leur sont proposées par l'enseignant (jouant alors le rôle de prescripteur), variable selon leurs connaissances et leur motivation du moment notamment. Une hypothèse forte de la double approche est que l'activité développée par un élève pour résoudre une tâche est un vecteur d'apprentissage potentiel (Robert \& Rogalski, 2002). La réalisation de la tâche constitue 
l'activité productive dans laquelle l'élève transforme le réel, qui devrait s'accompagner d'un apprentissage, la transformation de l'élève lui-même, la restructuration de ses connaissances, l'activité constructive (Pastré, 2007). La réalisation de la tâche ne signifie pas nécessairement qu'il y a apprentissage ou restructuration de connaissances, l'aide de l'enseignant peut jouer un rôle important.

La double approche didactique et ergonomique propose de considérer les pratiques enseignantes comme constituées de cinq composantes. Dans la présentation des composantes qui suit nous avons intégré les spécifications propres à l'enseignement de la chimie ${ }^{1}$, ces composantes des pratiques ayant initialement été définies pour l'enseignement des mathématiques (Vandebrouck, 2013).

Les deux premières composantes, cognitive et médiative, visent à caractériser les activités possibles ou effectives ${ }^{2}$ que l'enseignant suscite chez les élèves. La composante cognitive s'apprécie en étudiant les tâches prévues pour les élèves lors d'une séance et les contenus de chimie ou de physique qu'elles mettent en œuvre. La composante médiative englobe les choix d'organisation du travail des élèves dans la classe, l'accompagnement procuré par l'enseignant pendant le déroulement en classe (Robert, 2012). L'enseignant joue le rôle d'un médiateur entre l'élève et le savoir. Cet accompagnement peut être caractérisé par l'enrôlement dans la tâche, les aides apportées, l'identification du savoir mis en jeu (Rogalski, 2012) qui sont des caractéristiques d'une fonction d'étayage, au sens de Bruner (1996).

Trois autres composantes rassemblent des contraintes extérieures à la classe qui influent sur la séance et sur les composantes cognitive et médiative des pratiques. Masselot et Robert (2007) parlent de déterminants du métier ou des pratiques pour rendre compte des contraintes extérieures à la classe qui ont un effet sur ce qui se passe en classe et sur la préparation de la classe. La composante institutionnelle se rapporte à la prise en compte par l'enseignant du programme, des horaires, des contraintes institutionnelles, des ressources à sa disposition comme le matériel et les produits chimiques disponibles dans l'établissement (ressources spécifiques à cette discipline). La composante sociale concerne les relations que l'enseignant entretient avec ses collègues de travail, les préparateurs (autre spécificité de cette discipline par rapport aux mathématiques). Elle porte sur sa participation aux choix collectifs des collè- gues de l'établissement, sur les habitudes professionnelles des enseignants de physique-chimie, ce qui se rapproche du genre professionnel évoqué par Robert (2004) et Goigoux (2007) reprenant le concept introduit par Clot (1999). Les répercussions que le milieu social des élèves peut avoir sur le déroulement ou la conception de la séance font partie également de cette composante sociale (Butlen, Peltier-Barbier, \& Pézard, 2002). Enfin la composante personnelle inclut les représentations et connaissances de l'enseignant sur la chimie (ou la physique s'agissant d'enseignant de physique-chimie), ses convictions personnelles sur l'enseignement de la chimie, l'impact de son histoire personnelle sur son travail c'està-dire son expérience professionnelle. Ce découpage en cinq composantes vise à éclairer selon différents points de vue la complexité des pratiques de l'enseignant. Les composantes sont en fait imbriquées (Robert, 2012), leur recombinaison permet d'approcher les logiques d'action de l'enseignant.

Dans la mesure où nous nous intéressons par la suite à l'activité enseignante (un sous-ensemble des pratiques), par commodité nous parlerons des composantes de l'activité enseignante qui pour nous sont les composantes des pratiques. Les composantes des pratiques ont été définies à partir de l'observation et de l'analyse de nombreuses activités d'enseignants (Robert, 2008a; Robert \& Rogalski, 2002; Vandebrouck, 2013). Elles rassemblent les caractéristiques générales pertinentes qui dans ce cadre permettent de parvenir à l'objectif recherché, décrire et analyser les pratiques pour les comprendre tout en rendant compte de leur complexité. Lorsqu'on étudie l'activité d'un enseignant, il s'agit d'une partie de ses pratiques, ce qui légitime pleinement de conserver les mêmes critères d'analyse que pour les pratiques qui concernent une échelle plus globale.

\section{Analyse des tâches et de leur réalisation}

La méthodologie associée à ce cadre théorique consiste à analyser a priori les tâches que l'enseignant prévoit pour les élèves, c'est-à-dire sans tenir compte de leur réalisation en classe. Cette analyse est possible à partir de textes, d'exercices ou de protocoles de TP par exemple. Lorsqu'on ne dispose pas d'écrits destinés aux élèves, cette analyse a priori peut s'effectuer à partir des consignes données par l'enseignant durant la séance. La difficulté réside alors 
dans la part à faire entre ce qui relève des choix antérieurs à la réalisation de la séance et ceux provenant d'adaptations nécessitées par les actes des uns et des autres. Ensuite le déroulement de la séance de classe est étudié pour pointer l'organisation du travail en classe, l'étayage de l'activité des élèves. Les analyses du projet de séance et du déroulement de la séance informent principalement sur les composantes cognitive et médiative, et ne permettent pas d'accéder à l'intégralité des trois autres composantes, institutionnelle, sociale et personnelle. Pour cela d'autres données sont nécessaires telles que des entretiens pour accéder aux raisons et aux motifs de l'enseignant par exemple, ou des informations sur le contexte, l'établissement, etc.

Suivant ce qui a été effectué en didactique des mathématiques (Robert, 2008b), l'analyse des tâches en didactique de la chimie (ou de la physique) s'attache à déterminer si les élèves doivent mobiliser des connaissances, des savoir-faire anciens ou nouveaux, les adapter, lors de tâches simples ou plus complexes pour atteindre le but fixé. Une tâche simple met en jeu une connaissance ou un savoir-faire cité dans sa formulation. La réalisation d'une tâche plus complexe nécessite des connaissances, des savoirfaire et une réflexion conduisant à la mise en ouvre d'étapes qui ne sont pas précisées dans la formulation de la tâche. Le type d'aide fournie par l'enseignant peut alors s'avérer déterminant lors d'une résolution en classe, individuelle ou collective, et modifie les conditions de réalisation de la tâche.

\section{CONTEXTE DE L'ÉTUDE}

En France, les enseignants de physique-chimie enseignent deux disciplines, la physique et la chimie, qui sont institutionnellement reconnues comme expérimentales. Cela se traduit par deux modalités, l'enseignement en classe entière ${ }^{3}$, le " cours ", et en demi-groupe en salle spécialisée dite de travaux pratiques, le «TP ». Notre étude concerne la première séance réalisée en salle de TP sur les piles électrochimiques en terminale $S$, dernière classe du cursus scientifique des lycées généraux français. Le choix de ce thème est argumenté par la suite. À la fin de l'année les élèves passent le baccalauréat, examen national, sésame pour entreprendre des études supérieures mais aussi source de contraintes institutionnelles et sociales fortes pour les enseignants.

\section{Le programme et les savoirs associés}

Les séances observées sont des séances ordinaires, portant sur les piles au programme de la classe de terminale S (Ministère, 2001). Le programme assez détaillé, propose une progression en chapitres et des activités expérimentales introductives ou en application des notions abordées dans les différents chapitres.

Les piles se situent dans la troisième partie du programme de chimie (Ministère, 2001), qui traite de la prévision du sens d'évolution spontanée d'un système chimique, juste après l'introduction du critère d'évolution spontanée. Connaissant l'équation de réaction susceptible de rendre compte de l'évolution potentielle d'un système chimique, ce critère consiste à comparer le quotient de réaction ( $\mathrm{Qr}$, une fonction des concentrations des solutés du système intervenant dans l'équation de réaction) à la constante d'équilibre $\mathrm{K}$, une fonction de la température uniquement, attachée à l'équation de réaction. Un système tend à évoluer dans le sens (relativement à l'équation de réaction) tel que la valeur de $\mathrm{Qr}$ devient égale à celle de $\mathrm{K}$ à la température considérée. Pour déterminer le sens d'évolution d'un système, il faut calculer la valeur du quotient de réaction dans l'état considéré, la comparer à celle de la constante d'équilibre et conclure. Si le quotient de réaction est inférieur à la constante d'équilibre alors le sens prévu est le sens direct de l'équation de réaction, si le quotient est supérieur, il s'agit du sens inverse et enfin si le quotient de réaction est égal à la constante d'équilibre, le système est à l'équilibre chimique et n'évolue pas (la composition du système reste constante).

Les piles sont présentées comme des « dispositifs mettant en jeu des transformations spontanées permettant de récupérer de l'énergie » (Ministère, 2001, p. 100). Le premier contenu préconisé s'intitule " transferts spontanés d'électrons entre des espèces chimiques (mélangées ou séparées) de deux couples oxydant-réducteur du type ion métallique / métal $\mathrm{M}^{\mathrm{n}+} / \mathrm{M}(\mathrm{s}) »$ (ibid.). Le programme cite des exemples de piles pouvant être étudiées comme la pile Daniell, à l'aide d'un ampèremètre pour mettre en évidence le sens du courant et d'un voltmètre pour la f.é.m. « Utiliser le critère d'évolution spontanée pour déterminer le sens de déplacement des porteurs de charges dans une pile » est l'une des compétences exigibles. 
Le type de pile électrochimique au programme est constitué d'un premier ensemble de deux espèces chimiques (un oxydant et un réducteur conjugués), un soluté et un solide métallique, relié à un second ensemble de deux espèces oxydant-réducteur conjugués. Les solutions sont reliées par un pont électrochimique (dispositif contenant une solution ionique concentrée) qui permet le mouvement d'ensemble des ions, les solides métalliques par des conducteurs électriques (fil et résistors) dans lesquels circulent les électrons ${ }^{4}$. Le mouvement d'ensemble de ces différents porteurs de charge, électrons et ions, constitue un courant électrique délivré par la pile lorsqu'elle fonctionne. Ce mouvement est rendu possible lorsqu'on sépare l'oxydant du premier couple du réducteur du second. Si ce n'est pas le cas, il n'y a qu'une transformation chimique spontanée sans génération de courant électrique.

\section{Analyse du savoir}

Lors d'une étude précédente, la mise en œuvre du critère d'évolution a fait l'objet d'une analyse épistémologique, qui a mis en évidence les relations entre le niveau expérimental et celui du modèle. Ces liens ont été regroupés dans un schéma dit de fonctionnement du modèle thermodynamique (Kermen \& Méheut, 2011). Ce schéma visualise les différentes étapes du raisonnement mené lors de l'application du critère pour prévoir ou justifier des transforma- tions chimiques. Il a été utilisé pour catégoriser les réponses d'élèves à des questions nécessitant la mise en œuvre de ce modèle thermodynamique (Kermen \& Méheut, 2011) pour prévoir des transformations chimiques à partir d'une description d'un système initial.

Dans la présente étude, nous transformons cet outil, le schéma de fonctionnement du modèle thermodynamique, en un schéma de présentation des étapes du raisonnement permettant de visualiser les liens faits ou non entre les deux niveaux et les liens au sein d'un même niveau (Fig. 1), dans le cas des espèces intervenant dans la pile Daniell. Au schéma nous ajoutons l'interprétation de la réaction d'oxydoréduction par un transfert d'électrons (M4, Fig. 1). Il est usuel de représenter formellement ce transfert d'électrons entre le réducteur d'un couple et l'oxydant d'un autre par des demi-équations électroniques qui symbolisent le passage d'une espèce chimique à son espèce conjuguée par perte ou gain d'électrons. Ceci constitue une adaptation du schéma de fonctionnement. Dans la mesure où lors de l'observation des séances nous avons constaté que les enseignants mettaient en œuvre le critère d'évolution pour une transformation chimique d'oxydoréduction, ce schéma nous a paru être un outil pertinent pour décrire les sous-épisodes de la séance (la réalisation des sous-tâches) et pour pointer les va-et-vient entre modèle et niveau expérimental qu'il s'agit de ne pas confondre (enjeu récurrent de l'enseignement de la chimie).

Figure 1.

Étapes du raisonnement utilisant le critère d'évolution

\begin{tabular}{|c|c|c|c|c|c|c|}
\hline $\begin{array}{l}\text { Niveau } \\
\text { du } \\
\text { modèle }\end{array}$ & $\begin{array}{l}\text { Équation de réaction } \\
\mathrm{Cu}_{(\text {(aq) }}^{22}+\mathrm{Zn}_{(\mathrm{s})}=\mathrm{Zn}_{\text {(aq) }}^{2+}+\mathrm{Cu}_{(\mathrm{s})} \\
\mathrm{M} 1\end{array}$ & \multicolumn{2}{|c|}{$\begin{array}{l}\mathrm{Q}_{\mathrm{r}}=\frac{\left[\mathrm{Zn}^{2+}\right]}{\left[\mathrm{Cu}^{2+}\right]} \\
\text { Quotient de réaction, } \\
\text { valeur dans l'état } \\
\mathrm{M} 2 \quad \text { initial }: \mathrm{Q}_{\mathrm{r}, \mathrm{i}}\end{array}$} & \multicolumn{2}{|c|}{$\begin{array}{l}\text { Comparaison de } Q_{r, i} \\
\text { à K constante d'équi- } \\
\text { libre }=>\text { sens } \\
\text { d'évolution } \\
\text { M3 }\end{array}$} & $\begin{array}{l}\text { Transfert d'électrons } \\
\mathrm{Zn}_{(\mathrm{s})}=\mathrm{Zn}^{2+}{ }_{(\mathrm{aq})}+2 \mathrm{e}^{-} \\
\mathrm{Cu}^{2+}{ }_{(\mathrm{aq})}+2 \mathrm{e}^{-}=\mathrm{Cu}_{(\mathrm{s})} \\
\text { M4 }\end{array}$ \\
\hline \multirow[t]{2}{*}{$\begin{array}{c}\text { Niveau } \\
\text { expérimental }\end{array}$} & \multicolumn{2}{|c|}{$\begin{array}{l}\text { Description de l'état } \\
\text { initial du système : nom des } \\
\text { espèces chimiques, formules } \\
\text { chimiques, états physiques, } \\
\text { quantité de matière } \\
\text { E1 }\end{array}$} & \multicolumn{2}{|c|}{$\begin{array}{l}\text { Description de l'état final du } \\
\text { système : nom des } \\
\text { espèces chimiques, formules } \\
\text { chimiques, états physiques, } \\
\text { quantité de matière } \\
\text { E2 }\end{array}$} & & $\begin{array}{l}\text { Identification de la } \\
\text { sformation chimique (des } \\
\text { tifs et produits) : mise en } \\
\text { ion des deux descriptions } \\
\text { chimiques }\end{array}$ \\
\hline & \multicolumn{6}{|c|}{$\begin{array}{l}\text { Données expérimentales : liquide, solide, couleurs... pH, conductivité... } \\
\text { pression, température, correspondant à une description ordinaire }\end{array}$} \\
\hline
\end{tabular}


Dans la figure 1, nous présentons les étapes possibles d'un raisonnement utilisant le critère d'évolution, les contenus de chaque étape étant synthétisés dans un rectangle. Le niveau expérimental comporte quatre rectangles, le niveau du modèle également. Pour ne pas surcharger la figure nous n'avons pas mis toutes les flèches susceptibles de représenter les liens faits entre ces rectangles.

Le niveau expérimental est divisé en deux parties, l'une correspond à une description en termes d'objets et d'événements (couleur, formation de solide, etc.), une description ordinaire, l'autre à une description chimique en termes d'espèces chimiques (pour une justification de ces deux parties voir Kermen \& Méheut, 2011).

Si l'état initial seulement est connu (E1, Fig. 1), alors la confrontation avec le niveau du modèle, en l'occurrence l'équation chimique (M1, Fig. 1) permet de dire si a priori deux sens d'évolution sont possibles (lorsque les quatre espèces chimiques sont présentes) ou un seul (si deux espèces chimiques sont présentes seulement). L'expression du quotient de réaction (M2, Fig. 1) s'effectue en consultant l'équation de réaction (M1, Fig. 1), son calcul en regardant la composition de l'état initial (E1, Fig. 1). Puis la comparaison avec la constante d'équilibre (M3, Fig. 1) donne le sens d'évolution du système. En regardant à nouveau la composition de l'état initial (E1, Fig. 1) la connaissance du sens d'évolution permet de décrire qualitativement l'état final (E2, Fig. 1) et d'identifier la transformation chimique attendue (E3, Fig. 1).

Si l'état final est connu (E2, Fig. 1), alors l'identification d'une transformation chimique (E3, Fig. 1) est possible en comparant avec la description de l'état initial (E1, Fig. 1) qui doit mettre en évidence un changement d'espèces chimiques. L'écriture de l'équation chimique est alors possible (M1, Fig. 1) et l'application du critère d'évolution (voir ci-dessus) vise à vérifier que l'évolution constatée est conforme.

L'interprétation en termes de transfert d'électrons (M4, Fig. 1) peut se faire une fois l'évolution connue que ce soit après mise en œuvre du critère d'évolution ou après observations et écriture de l'équation chimique simplement (M1, Fig. 1).

\section{ProblématiQue}

Notre projet à moyen terme vise à documenter les pratiques des enseignants de physique-chimie pour les comprendre (recherche de signification) et pour tenter de les expliquer (recherche des raisons des choix et mise au jour des contraintes). Cela passe par des observations de plusieurs enseignants, ce que nous nous attachons à faire ici. Envisager l'échelle globale consiste à les observer sur des périodes qui ne soient pas trop brèves pour aborder l'analyse des scénarios d'ensemble sur plusieurs mois (Masselot \& Robert, 2007). L'objectif de cette étude est la comparaison d'une même séance chez trois enseignantes pour effectuer des mises en relation permettant de repérer d'éventuelles régularités et des éléments de variation interindividuelle et intra-individuelle de leur activité en classe. Il s'agit donc d'une étude locale. Les choix et actions de l'enseignante qui suscitent les activités des élèves dont nous percevons les traces, constituent notre objet d'étude. Nos analyses visent à révéler les choix et raisons des enseignants et à les attribuer aux différentes composantes des pratiques. Choisir un même thème permet de caractériser la façon dont chaque enseignante s'approprie le programme, ressent les contraintes institutionnelles ${ }^{5}$ qu'il véhicule et traduit cela dans la conception de la séance et dans la mise en œuvre pour les élèves particuliers qu'elle a en face d'elle. Il est alors possible de retrouver l'imbrication de plusieurs composantes des pratiques ou de révéler l'existence de tensions entre ces composantes (Kermen \& Colin, 2014). En comparant le déroulement des deux séances d'une même classe pour une même enseignante, on peut identifier des variabilités susceptibles d'être attribuées aux comportements des élèves puisque les contraintes et le projet de séance sont les mêmes.

Nous avons choisi d'observer des séances de TP parce qu'a priori l'effectif étant diminué par rapport à celui d'une séance en classe entière, elles permettent aux élèves d'interagir plus facilement avec l'enseignante (c'est une supposition de notre part). Par ailleurs on peut attendre davantage d'engagement des élèves puisque des tâches expérimentales précises leur sont proposées avec en général des questions de réflexion, ce qui est un peu moins fréquent en classe entière où on peut imaginer que le nombre plus élevé d'élèves incite certains d'entre eux à une activité minimale d'écoute. 
Le choix du thème piles électrochimiques est intentionnel, parce que ce thème est à la croisée des deux disciplines chimie et physique, et concerne plus précisément, transformations chimiques spontanées et générateur électrique. Il peut être révélateur de liens faits ou non par les enseignantes (Kermen \& Colin, 2014). De plus lier le critère d'évolution spontanée d'un système chimique à la compréhension du principe de fonctionnement d'une pile électrochimique constituait une nouveauté dans l'enseignement secondaire français, qu'il est donc intéressant d'étudier. Le programme actuel (Ministère, 2011) ne mentionne pas ces concepts (quotient de réaction et critère d'évolution), une étude les incluant ne peut donc plus être menée.

Dans cet article nous nous centrons sur l'analyse de la première partie de la séance, où une transformation chimique spontanée est réalisée en préalable à la constitution de piles électrochimiques dans la suite de la séance, suite qui a déjà fait l'objet d'une publication (Kermen \& Colin, 2014). La réalisation d'une transformation chimique et son interprétation ou sa prévision dans une salle de TP mêlent nécessairement des aspects expérimentaux et des aspects relevant du niveau des modèles, aspects qu'il est important de distinguer et mettre en relation à bon escient pour un apprentissage cohérent. Le passage de l'étude d'une transformation chimique spontanée à celui d'une pile nécessite une interprétation au niveau des modèles. Tout type de transformation chimique peut-il «servir » à la conception d'une pile? Comment utiliser cette spontanéité de l'évolution d'un système chimique pour constituer une pile? Ces questions se résolvent en faisant le lien entre le courant électrique fourni par une pile et la transformation chimique spontanée étudiée. La spontanéité indique que les espèces réagissent (plus ou moins rapidement) lorsqu'on les met au contact. Un courant électrique s'interprète comme un mouvement d'ensemble de porteurs de charge, qui sont des électrons dans les conducteurs électroniques, et des ions dans les solutions. Il faut donc « disposer » d'une transformation chimique qui s'interprète avec un transfert d'électrons entre espèces chimiques (d'un réducteur vers un oxydant), transformation à laquelle on associe une réaction d'oxydoréduction. Il s'agit ensuite de déterminer comment rendre ce transfert d'électrons possible dans un circuit électrique pour obtenir un courant électrique. Ces ques- tions et la façon de les résoudre sont au cour de la première partie des séances observées.

Une comparaison des projets de séance par le biais d'une analyse des fiches TP et des séances effectivement réalisées permet d'apprécier les choix préalables et les choix et adaptations dans l'action effectués par les enseignantes. La diversité de ces choix ou au contraire leur similarité fourniront des éléments de variabilité ou des régularités.

Nos analyses visent à répondre à deux séries de questions de recherche. La première série concerne la façon dont les enseignantes mettent en scène le contenu traité dans les séances pour susciter l'activité des élèves. Ces questions de recherche ont été précisées au fur et à mesure de l'analyse des séances de classe pour caractériser les différences et points communs. Les trois enseignantes s'appuient sur une transformation chimique interprétée par la même équation de réaction mais pas tout à fait sur le même dispositif expérimental ni sur le même questionnement. Il s'agit d'examiner comment la transformation chimique est utilisée pour amener les élèves à l'idée de pile (de transfert d'électrons). La caractérisation de la transformation chimique elle-même est liée aux observations possibles, son interprétation s'effectue par l'écriture d'une équation de réaction. Selon la part attribuée au niveau des modèles et au niveau expérimental, le rôle de l'un ou de l'autre peut être accentué. Des différences de mise en œuvre et de questionnement peuvent conduire à des activités d'élèves dissemblables. La seconde série de questions vise à caractériser le travail des enseignantes en recherchant des régularités et des variations et en reconstruisant leurs logiques d'action (ou logiques d'enseignement) en termes d'imbrications et, ou tensions entre composantes de leur activité pour comprendre les choix effectués.

Première série de questions de recherche : - Comment les enseignantes font-elles pour passer d'une transformation chimique spontanée à l'idée de pile? Autrement dit, quand la question des transferts d'électrons apparaitelle dans le déroulement de la séance et comment est-elle utilisée?

- Comment le choix d'un dispositif expérimental pour réaliser une transformation chimique et celui du questionnement associé influent-il sur le déroulement de chaque séance et sur l'activité des élèves? En particulier les observations conditionnent-elles ou pas l'écriture d'une équation de réaction? 
Isabelle Kermen \& Philippe Colin

- Quels rôles respectifs chaque enseignante attribue-t-elle au niveau expérimental et au niveau des modèles?

Seconde série de questions de recherche:

- Quelles régularités et quelle variabilité interpersonnelle et intra-individuelle, la conception et la mise en œuvre de ces séances révèlent-elles?

- Quelle interprétation de l'activité de ces enseignantes en termes de composantes des pratiques et quelles logiques d'action peut-on proposer?

Nous exposons maintenant la méthodologie mise en œuvre pour répondre à ces questions.

\section{MÉTHODOLOGIE DE RECUEIL ET D'ANALYSE}

\section{Recueil des données}

Nous avons observé et filmé les séances de trois enseignantes au mois de mars 2012 dans trois lycées. Il s'agit de séances ordinaires dans la mesure où les enseignantes ont mis en place leur propre projet d'enseignement sur lequel nous n'avons eu aucun regard préalable, ni a posteriori d'ailleurs. Ces trois enseignantes sont désignées par des pseudonymes. Ce sont des enseignantes expérimentées ayant entre 10 et 30 ans d'ancienneté, et plus de 4 ans d'enseignement de ce programme. Chaque enseignante porte un micro-cravate permettant de suivre les discussions qu'elle a pu avoir avec des binômes d'élèves lors de ses déplacements. Nous disposons des enregistrements des séances des deux groupes des classes (effectif supérieur à 24) de mesdames Branly et Forest et d'un seul pour madame Say puisque sa classe comportait moins de 24 élèves. Chacune d'entre elles avait déjà été filmée lors d'autres séances. On peut donc penser que la présence de la caméra et des observateurs en fond de salle de classe n'a que peu perturbé le déroulement des séances.

Chaque enseignante a été brièvement interrogée juste après la séance sur son ressenti, l'atteinte de ses objectifs, et également pour déterminer la place de la séance observée dans sa progression. Puis quelques semaines ou mois plus tard nous avons organisé un nouvel entretien de l'enseignante devant sa vidéo de classe. Pour ce deuxième entretien, nous avons dit à chaque enseignante que notre objectif était de comprendre comment elle travaille, que ce travail prenait en compte l'apprentissage des élèves, le fait de travailler dans un établissement, de suivre un programme par exemple. Et nous avons demandé à chacune de commenter ce qu'elle voyait et entendait, si elle le jugeait nécessaire. Nous leur avons précisé que nous ne poserions qu'une ou deux questions pour préciser éventuellement certains aspects qu'elles auraient abordés ou si cela s'avérait nécessaire pour mieux comprendre le sens de leur propos. Elles avaient pour consigne de stopper le déroulement de la vidéo à ce moment-là pour que leurs commentaires soient bien audibles. Ceux-ci sont parfois allés bien au-delà de la séance observée (réflexion sur les futurs programmes par exemple), mais pour certains passages sujets à questionnement de notre part, il n'y en a pas eu. En effet, ces entretiens visent à mettre l'enseignante dans la position d'un professionnel qui commente sa pratique et non pas dans celle d'un professionnel jugé et critiqué, tout en essayant d'accéder aux raisons de ses choix. Nous n'avons posé de questions que pour préciser un thème discuté par l'enseignante elle-même. Cet aspect limite la portée de cet entretien et des analyses que nous en tirons.

Les données sont donc constituées des enregistrements audio des entretiens, des enregistrements vidéo des séances de classe et des fiches TP distribuées aux élèves en début de séance par les trois enseignantes.

\section{Traitement et analyse des données}

Les enregistrements ont été intégralement transcrits pour être analysés. Les instructions et questions figurant dans la fiche TP ont été converties en tâches attendues de la part des élèves, mobilisant tel ou tel contenu, à partir de notre réflexion sur ce programme (Kermen \& Méheut, 2008). La description d'un dispositif expérimental et des instructions qui l'accompagnent appelle un suivi du protocole mettant en jeu divers savoir-faire expérimentaux. Lorsqu'une question porte sur l'interprétation des phénomènes, nous indiquons les connaissances et concepts pouvant être mobilisés et selon quelle logique. En fait nous inférons les activités possibles des élèves compte tenu de nos connaissances de leurs raisonnements et difficultés habituels.

Nous avons confronté le résultat de l'analyse de la fiche TP, qui a délimité des tâches et des soustâches, aux transcriptions des enregistrements, ce qui a conduit à un découpage en épisode et sous- 
épisodes, chaque (sous-)épisode correspondant à la réalisation d'une (sous-)tâche prévue dans l'analyse a priori. Notre analyse se restreignant à la première partie de la séance, nous avons procédé à une analyse fine du déroulement en prenant appui sur le fonctionnement du modèle thermodynamique pour repérer la réalisation des sous-tâches (cf. paragr. Analyse du savoir).

Nous avons ensuite juxtaposé les transcriptions des déroulements des deux groupes d'une même enseignante, en mettant en regard la réalisation des sous-tâches identiques afin de dégager des éléments de stabilité et de variabilité. Le discours de chaque enseignante et les échanges avec les élèves ont fait l'objet d'une attention particulière.

L'examen des transcriptions des entretiens avec les enseignantes a permis de compléter les analyses de chaque déroulement en recherchant les commentaires faits sur les choix de tâches effectués et sur la façon dont la première partie des séances a été vécue.

\section{ANALYSE DIDACTIQUE DE LA PREMIÈRE PARTIE DES FICHES TP}

Nous avons effectué une analyse didactique des fiches TP distribuées par les enseignantes, pour répertorier les contenus abordés, les tâches attendues des élèves, pointer les difficultés possibles et inférer une organisation possible du travail en classe.

\section{Des caractéristiques communes}

Les trois fiches TP présentent une structure commune quant à l'ordre des contenus abordés, conforme à ce qui figure dans le programme (Ministère, 2001). La première partie concerne une transformation chimique pour laquelle il est fait mention de « transfert direct d'électrons » et mettant en jeu les espèces chimiques qui constituent la pile Daniell. La deuxième partie propose de réaliser une pile Daniell et d'étudier le courant fourni dans un circuit simple puis la tension à vide aux bornes de la pile. L'objectif qui transparaît dans les questions posées est de relier le sens du courant à l'évolution constatée dans la transformation chimique effectuée préalablement. Ces fiches abordent ensuite la dépendance de la force électromotrice d'une pile selon un ou plusieurs facteurs, nature des couples oxydant- réducteur, concentration des espèces chimiques. À la lecture des fiches, il se dégage des modalités de travail communes : on attend des élèves un suivi du protocole décrit consistant, selon les expériences, à faire des mesures ou/et des observations, on peut s'attendre à des phases de recherche en autonomie pour répondre aux questions posées, et probablement à des phases collectives de réponses avec un guidage adapté au degré de complexité de la question.

Ces fiches ont également en commun de ne pas comporter de préambule mentionnant les objectifs poursuivis, ni de questionner le passage du mélange des espèces chimiques donnant lieu à la transformation chimique initiale à une pile. En effet il n'est pas demandé d'imaginer un dispositif expérimental permettant d'obtenir un courant électrique, ni de protocole pour tester son fonctionnement. Le dispositif expérimental consistant à séparer les espèces d'une certaine façon (métal cuivre avec ions cuivre, et métal zinc avec ions zinc) est fourni, les expériences à faire sont décrites.

Lien entre niveau expérimental et niveau du modèle : deux approches différentes

Les premières parties des fiches traitent toutes d'une expérience conduisant au même résultat, formation de métal cuivre et d'ions zinc, à partir de deux compositions initiales différentes (cf. paragr. Des variantes autour d'une transformation chimique) et selon deux approches: dans l'une, une fois l'expérience effectuée, les observations conduisent à caractériser la transformation chimique justifiée a posteriori par l'application du critère d'évolution (niveau du modèle) tandis que dans l'autre l'utilisation du modèle permet la prévision d'une transformation chimique qui est ensuite validée expérimentalement (fiche de madame Forest). Quelle que soit l'approche adoptée, aucune des fiches ne détaille l'application du critère d'évolution (voir paragr. Des observations à l'identification de la transformation chimique et Du modèle à l'identification de la transformation chimique pour madame Forest), alors qu'elle s'effectue en plusieurs étapes et nécessite l'introduction d'une grandeur, le quotient de réaction, non mentionnée dans l'énoncé. Cette application qui constitue l'activité possible des élèves, correspond à des va-et-vient entre le niveau du modèle et la situation expérimentale (voir paragr. Analyse du savoir). 
L'application du critère d'évolution est une tâche qui correspond à une compétence exigible pour l'examen du baccalauréat et à un raisonnement enseigné dans le précédent chapitre de chimie (traité plusieurs semaines auparavant par chaque enseignante), dont les enseignantes considèrent vraisemblablement qu'il doit être à terme connu des élèves, ce qui peut expliquer qu'il ne soit pas détaillé. Cependant, dans les sujets de baccalauréat et les exercices des manuels, ce raisonnement est toujours guidé par plusieurs questions indiquant les différentes étapes et grandeurs nécessaires.

\section{Des variantes autour d'une transformation chimique}

Le tableau 1 donne une vue d'ensemble du contenu des fiches, en regroupant les informations qu'elles comportent et qui permettent de mener à bien les tâches assignées aux élèves.

La fiche de madame Branly et celle de madame Forest utilisent la même expérience mettant en jeu initialement les quatre espèces chimiques, donc il y a a priori deux sens d'évolution potentiels. Cette expérience ne diffère que par l'aspect physique du cuivre, et la présence ou non d'un thermomètre. L'expérience figurant dans la fiche de madame Branly est identique à celle du document d'accompagnement rédigé par les auteurs du programme. Par contre celle de madame Say ne met en jeu que deux espèces réac- tives, une fois celles-ci identifiées par les élèves, le sens d'évolution est connu.

\section{Des observations à l'identification de la transformation chimique : deux approches contrastées}

Comme indiqué plus haut, mesdames Branly et Say ont adopté la même approche : vérifier que les observations sont compatibles avec l'application du critère d'évolution. Alors que l'on aurait pu s'attendre à des tâches relativement proches conduisant à des scénarios voisins, l'analyse des fiches TP des deux enseignantes montre que leurs approches sont en fait très contrastées.

\section{Des difficultés d'observations prévisibles pour l'expérience de madame Branly}

Le premier paragraphe de la fiche de madame Branly commence par une phrase qui peut être comprise comme décrivant l'objectif à atteindre : « mise en évidence expérimentale d'une transformation chimique spontanée par transfert direct d'électrons », un peu ambigüe. Cela peut laisser penser que le transfert direct d'électrons constitue la mise en évidence expérimentale au lieu de son interprétation. Immédiatement après les formules des couples oxydant-réducteur concernés sont indiquées. Ensuite

Tableau 1.

Variantes autour d'une transformation chimique

\begin{tabular}{|c|c|c|c|}
\hline & Madame Branly & Madame Forest & Madame Say \\
\hline Nombre initial d'espèces chimiques réactives & 4 & 4 & 2 \\
\hline Espèces chimiques solides & $\begin{array}{l}\text { Lame de zinc, poudre de } \\
\text { cuivre }\end{array}$ & $\begin{array}{l}\text { Lame de zinc, lame de } \\
\text { cuivre }\end{array}$ & Poudre de zinc \\
\hline Espèces chimiques solutés & \multicolumn{2}{|c|}{$\begin{array}{l}\text { Sulfate de cuivre (II) et sulfate de zinc, mêmes } \\
\text { concentrations, mêmes volumes }\end{array}$} & $\begin{array}{l}\text { Sulfate de cuivre } \\
\text { (II) }\end{array}$ \\
\hline Couples oxydant-réducteur fournis & oui & oui & non \\
\hline Équation chimique fournie & non & $\begin{array}{l}\text { oui, deux équations } \\
\text { inverses }\end{array}$ & non \\
\hline Constante d'équilibre fournie & oui & oui & oui \\
\hline Observations requises & $\begin{array}{l}\text { Température et couleur } \\
\text { de la solution }\end{array}$ & aucune & oui, sans précision \\
\hline Identification des ions zinc & Sans objet & Sans objet & oui \\
\hline Indication & & & Cinétique lente \\
\hline
\end{tabular}


Isabelle Kermen \& Philippe Colin

figure la liste du matériel et des produits à disposition, puis sous le sous-titre expérience, une suite d'instructions pour réaliser un mélange de quatre espèces chimiques (voir tableau 1). Il faut à l'aide d'un thermomètre noter l'évolution de la température du mélange, qu'il s'agit ensuite de filtrer pour observer la couleur du filtrat.

Dans les conditions expérimentales proposées, le dépôt rougeâtre sur la lame de zinc et l'affaiblissement de la couleur de la solution risquent de prendre un certain temps. Le transfert thermique avec l'environnement est de faible valeur, il est donc peu probable qu'une modification de la température soit constatée. Un élève peut alors se poser la question du moment où il faut cesser de mesurer la température et celle où il faut faire la filtration demandée. Une filtration précoce peut empêcher de voir les modifications attendues.

Deux questions structurent ensuite l'interprétation. La première question demande d'utiliser les observations pour écrire l'équation de la réaction associée à la transformation chimique du système.

Les élèves doivent traduire les observations (couleur et dépôt) en termes d'espèces chimiques, formation de cuivre et consommation d'ions cuivre, pour identifier en partie la transformation chimique (un réactif et un produit) et ensuite passer à l'interprétation en termes de réaction chimique et à son écriture symbolique. La donnée des couples oxydantréducteur oriente les élèves et leur indique qu'il faut prendre en compte le couple de l'élément zinc, alors que rien dans les observations ne permet de le voir. Il s'agit d'un réinvestissement d'une connaissance antérieure, l'écriture d'une équation de réaction d'oxydoréduction, les deux couples ayant été fournis.

La deuxième question, en donnant la valeur de la constante d'équilibre de l'équation, demande de montrer que le sens d'évolution du système prévu est compatible avec les observations, en appliquant le critère d'évolution (voir Fig. 1).
Une identification expérimentale des produits de la transformation au détriment d'une recherche effective du sens d'évolution pour madame Say

Le premier paragraphe de la fiche de madame Say comporte deux parties distinctes, expérience puis analyse. La partie expérience est composée de deux courts protocoles séparés par un large espace pour répondre.

Dans le premier protocole, les élèves doivent introduire dans deux tubes à essais une solution de sulfate de cuivre (II) et de la poudre de zinc seulement dans l'un des deux, avant d'agiter. L'enseignante attend des élèves qu'ils remarquent l'éclaircissement de la solution et un dépôt solide sur la poudre de zinc. Dans le deuxième protocole, il s'agit de filtrer le contenu du tube, récupérer le filtrat et y ajouter une solution d'hydroxyde de sodium goutte à goutte, puis en excès. Les élèves devront constater la formation d'un précipité blanchâtre (tout dépend s'il reste encore des ions cuivre en solution) puis sa disparition. Dans les deux cas, il est demandé de schématiser l'expérience et noter les observations, qui ne sont pas spécifiées.

La partie analyse permet l'interprétation de l'expérience par le biais de trois questions. La première " quelle est la transformation chimique (a) qui a eu lieu dans le tube 2 ?». Il faut identifier les espèces chimiques formées et consommées à partir des observations effectuées, mais le raisonnement à conduire n'est pas détaillé. Les élèves doivent prendre en compte les observations des deux expériences successives pour faire un bilan des espèces chimiques formées dans la première expérience. Le dépôt rougeâtre sur la poudre de zinc s'accompagne d'un éclaircissement de la solution, vu en comparant avec le premier tube, signifiant qu'il y a moins d'ions cuivre (II) en solution dans l'état final de la première expérience. Ces deux observations sont interprétées comme la conversion des ions cuivre (II) en métal cuivre.

Figure 2.

Description chimique partielle de la première expérience

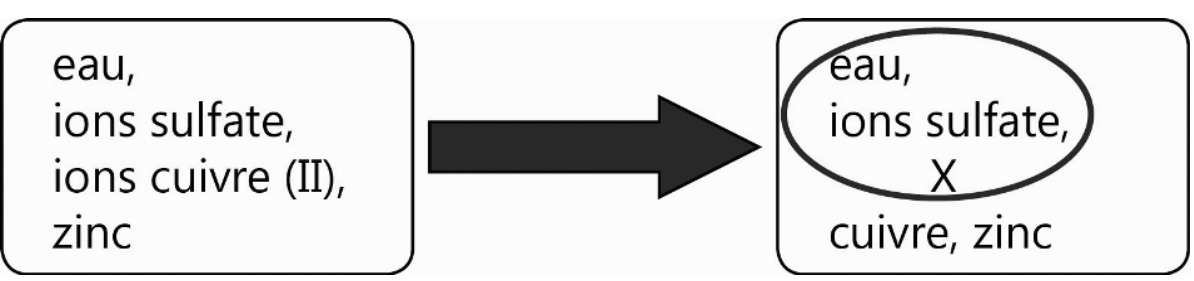


La seconde expérience vise à montrer la présence d'ions zinc dans la solution incolore du tube 2 (après filtration, solution entourée dans la figure 2). En effet les ions zinc (X) forment en présence d'ions hydroxyde un précipité blanc d'hydroxyde de zinc (Y) qui se dissout avec un excès d'ions hydroxyde pour former des ions tétrahydroxozincate (Z) (Fig 3). Comme le montre la figure 3 ci-dessous, les élèves sont confrontés à trois espèces chimiques inconnues, s'ils n'ont jamais rencontré cette expérience.

La présence d'ions zinc (dans l'état final de la critère d'évolution (voir paragr. Analyse du savoir, Fig. 1). Nous rappelons (voir paragr. Des variantes autour d'une transformation chimique) que la présence dans les conditions initiales de seulement deux espèces chimiques sur les quatre des deux couples oxydant-réducteur (voir tableau 1) fournit d'emblée la réponse au sens d'évolution dans le cadre d'un contrat didactique standard.

\section{Du modèle à l'identification de la}

Figure 3.

Description chimique partielle de l'expérience 2

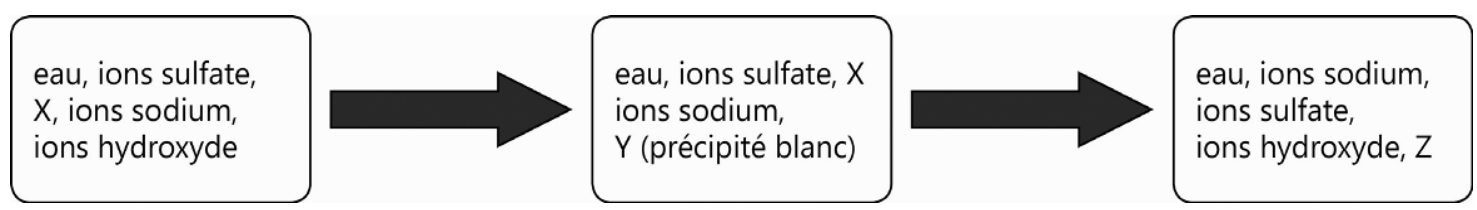

première expérience) s'interprète alors comme provenant de la modification du métal zinc qui a réagi avec les ions cuivre (II) pour donner des ions zinc et du métal cuivre. Les espèces présentes (dans l'état final de la première expérience) ne sont pas les mêmes qu'au début, il y a bien eu une transformation chimique (a) dont il est question dans l'énoncé. Les élèves ont à fournir une description chimique conduisant à identifier une transformation chimique parce que de nouvelles espèces chimiques ont été formées. Il n'est pas demandé d'écrire l'équation de réaction correspondante à ce stade.

La deuxième question est fermée "y a-t-il eu transfert d'électrons? si oui lequel? ». La réponse attendue permet de faire un lien avec le titre du paragraphe. L'examen des formules des espèces chimiques initialement présentes et des espèces formées permet de dire que des électrons ont été transférés du zinc aux ions cuivre (II), ce qui a formé des ions zinc et du métal cuivre. Il est aussi possible que les élèves s'appuient sur les demi-équations électroniques pour répondre.

La troisième question donne «la constante d'équilibre de l'équation (a) » et demande si « l'évolution du système chimique était prévisible ». Le raisonnement à conduire n'est pas fourni. Il faut en premier lieu écrire l'équation de réaction chimique associée à la transformation (a), puis appliquer le transformation chimique pour madame Forest

Madame Forest choisit de centrer son approche sur l'usage du modèle, les observations venant vérifier a posteriori le sens d'évolution prédit. Le premier paragraphe de la fiche de madame Forest commence avec une description d'expérience à réaliser. Il n'est pas dit ce qu'il faut faire avec le mélange obtenu (quatre espèces, voir tableau 1), il n'y a pas de demande d'observation. Juste après la description, sont indiqués les couples oxydant-réducteur, deux équations de réactions inverses ainsi que leur constante d'équilibre, suivis par une consigne «en exploitant l'une ou l'autre de ces équations, prévoir le sens d'évolution du système ». À la différence des deux autres enseignantes, la formulation adoptée ne lie pas les observations expérimentales et la prévision théorique demandée. Il est possible que les élèves soient surpris par la présence de deux équations de réaction, qu'ils ne sachent laquelle prendre. Comme dans les autres fiches, l'application du critère d'évolution, qui conduit à prévoir le sens d'évolution du système, n'est pas détaillée.

Ensuite dans un encadré la définition d'un transfert spontané d'électrons est présentée comme conclusion : " lorsqu'on met en contact le réducteur d'un couple et l'oxydant d'un autre couple, on peut observer un transfert spontané d'électrons du 
réducteur vers l'oxydant. Le transfert est direct si les électrons transitent du réducteur vers l'oxydant sans l'intermédiaire d'un autre conducteur métallique. » La formulation « autre conducteur métallique » peut sembler énigmatique car il n'est pas précisé que le réducteur peut être un métal. De plus elle comporte une maladresse, on «n'observe » pas « un transfert d'électrons », on l'infère d'observations expérimentales telles qu'un passage de courant. Ce sont des informations données aux élèves, appartenant au modèle, plus qu'une conclusion en lien avec la prévision du sens d'évolution.

\section{Synthèse de l'analyse didactique des fiches de TP}

Toutes ces fiches commencent par une transformation chimique mettant en jeu un mélange des espèces chimiques intervenant dans la pile Daniell, conformément aux suggestions du programme (explicitées dans le document d'accompagnement) et aux propositions qu'on trouve dans les manuels scolaires (Barde et al., 2002; Tomasino, 2002). Seule celle de madame Branly reprend in extenso le descriptif et le questionnement de la transformation chimique figurant dans le document d'accompagnement du programme. Toutes les fiches mentionnent un transfert direct d'électrons en introduction pour l'une, au milieu des analyses pour une autre et en conclusion pour la dernière.

Les différences en termes d'approche, plus précisément en termes de places respectives occupées par le niveau expérimental et le niveau du modèle (voir Fig. 1), demandent aux élèves un type de réflexion différent, centrée d'abord sur l'utilisation du modèle ou d'abord sur l'observation. Le dispositif expérimental (voir tableau 1) n'étant pas le même, l'observation seule ne suffit pas à définir la transformation chimique en jeu dans la fiche de madame Branly, tandis que dans celle de madame Say, l'observation fait appel à une connaissance que les élèves ne possèdent peut-être pas (s'ils n'ont jamais réalisé de précipité d'hydroxyde métallique).

Ces trois études a priori nous permettent de faire un premier bilan sur les composantes des pratiques révélées par l'analyse de l'activité de ces enseignantes. L'utilisation par les trois enseignantes du thème introductif proposé par le programme (utilisé à la lettre pour madame Branly) illustre l'influence forte de ce déterminant institutionnel. La diversité des approches et des tâches proposées, témoigne d'une composante cognitive différente où l'on perçoit la marque personnelle de chaque enseignante et qui résulte au moins en partie d'une perception différente de l'enseignement de la chimie. En effet dans l'entretien post-séance, madame Say souligne l'importance de bien marquer pour les élèves la distinction entre observations et analyse, ce qui explique la structure de sa fiche (les expériences nettement séparées de leur analyse), et donne une indication sur l'image qu'elle souhaite donner aux élèves, la chimie comme science expérimentale. Madame Forest présente l'originalité de proposer aux élèves de choisir l'équation de réaction à partir de laquelle ils doivent faire une prévision. Comment va-t-elle gérer un éventuel choix différent des élèves? Son objectif est-il de montrer que le sens d'évolution prévu dépend de l'équation choisie mais pas le résultat expérimental? Cela pourrait être une autre façon de découpler le niveau expérimental du niveau des modèles. Comme elle nous l'a déclaré dans l'entretien post-séance, elle souhaite qu'ils fassent un choix, et prennent conscience que, quel que soit celui-ci, ils vont aboutir à une conclusion qui sera la même.

\section{ANALYSE DES SÉANCES DE CLASSE}

Dans un premier temps nous présentons une analyse de la structure globale des déroulements de chaque séance. Puis les descriptifs de ces séances visualisés par les schémas de déroulement sont complétés par l'analyse des choix en lien avec nos questions de recherche.

\section{Structure globale des séances de classe}

La structure des déroulements est nettement différente tant par la succession des épisodes, ce que laissait présager l'analyse a priori des fiches TP, que par leur durée (cf. tableau 2 ci-dessous) et le mode de travail engagé (cf. tableau 3 ).

Seule madame Branly ne réserve pas de moment spécifiquement dédié à une réflexion individuelle des élèves, pour répondre à des questions ou pour conduire des expériences (tableau 3). Madame Forest change de mode de travail d'un groupe à l'autre pour la mise en commun, puisqu'elle n'envoie pas d'élève écrire la correction au tableau dans le groupe 2. 
Tableau 2.

Répartition temporelle des épisodes (en minutes et secondes)

\begin{tabular}{|c|c|c|c|c|c|c|}
\hline \multirow[t]{2}{*}{ Épisodes } & \multirow[t]{2}{*}{ Madame Say } & \multicolumn{2}{|c|}{ Madame Branly } & \multicolumn{2}{|c|}{ Madame Forest } & \\
\hline & & Groupe 1 & Groupe 2 & Groupe 1 & Groupe 2 & \\
\hline Introduction de la séance & $3: 30$ & $11: 22$ & $14: 27$ & $1: 30$ & $1: 45$ & \\
\hline Réalisation des expériences & $19: 14$ & $8: 38$ & $2: 55$ & $16: 24$ & $13: 23$ & $\begin{array}{l}\text { Prévision du sens } \\
\text { d'évolution }\end{array}$ \\
\hline $\begin{array}{l}\text { Institutionnalisation des } \\
\text { observations }\end{array}$ & $2: 58$ & $3: 02$ & $4: 28$ & $2: 56$ & $0: 55$ & $\begin{array}{l}\text { Lien avec les obser- } \\
\text { vations }\end{array}$ \\
\hline Réponses aux questions & $11: 33$ & 4:09 & $3: 42$ & $0: 48$ & $0: 25$ & Conclusion \\
\hline $\begin{array}{l}\text { Transition avec la tâche } \\
\text { suivante }\end{array}$ & $0: 42$ & $0: 51$ & aucune & $0: 28$ & $0: 47$ & \\
\hline
\end{tabular}

Tableau 3.

Formes de travail proposées aux élèves

\begin{tabular}{|c|c|c|c|c|c|c|}
\hline \multirow[t]{2}{*}{ Épisodes } & \multirow{2}{*}{$\begin{array}{l}\text { Madame } \\
\text { Say }\end{array}$} & \multicolumn{2}{|c|}{ Madame Branly } & \multicolumn{2}{|c|}{ Madame Forest } & \\
\hline & & Groupe 1 & Groupe 2 & Groupe 1 & Groupe 2 & \\
\hline $\begin{array}{l}\text { Introduction de la } \\
\text { séance }\end{array}$ & \multicolumn{3}{|c|}{$\begin{array}{l}\text { écouter et répondre aux questions dans un } \\
\text { dialogue collectif }\end{array}$} & \multicolumn{2}{|l|}{ écouter } & \\
\hline $\begin{array}{l}\text { Réalisation des expé- } \\
\text { riences }\end{array}$ & $\begin{array}{l}\text { manipuler } \\
\text { en binômes, } \\
\text { réfléchir }\end{array}$ & $\begin{array}{l}\text { écouter, } \\
\text { regarder l'ex- } \\
\text { périence faite } \\
\text { par un élève } \\
\text { au tableau } \\
\text { et copier le } \\
\text { tableau }\end{array}$ & $\begin{array}{l}\text { écouter, } \\
\text { regarder } \\
\text { l'expérience } \\
\text { faite par le } \\
\text { professeur } \\
\text { au tableau } \\
\text { et copier le } \\
\text { tableau }\end{array}$ & $\begin{array}{l}\text { réfléchir indi- } \\
\text { viduellement, } \\
\text { puis prendre } \\
\text { la correction } \\
\text { écrite faite } \\
\text { par un élève, } \\
\text { Léo, au } \\
\text { tableau }\end{array}$ & $\begin{array}{l}\text { réfléchir } \\
\text { individuel- } \\
\text { lement, puis } \\
\text { répondre aux } \\
\text { questions } \\
\text { et prendre } \\
\text { en note la } \\
\text { correction } \\
\text { orale }\end{array}$ & $\begin{array}{l}\text { Prévision du sens } \\
\text { d'évolution }\end{array}$ \\
\hline $\begin{array}{l}\text { Institutionnalisation } \\
\text { des observations }\end{array}$ & \multicolumn{3}{|c|}{$\begin{array}{l}\text { écouter et répondre aux questions, copier } \\
\text { les inscriptions du tableau }\end{array}$} & $\begin{array}{l}\text { écouter, } \\
\text { regarder } \\
\text { l'expérience } \\
\text { faite par le } \\
\text { professeur et } \\
\text { répondre aux } \\
\text { questions }\end{array}$ & $\begin{array}{l}\text { écouter, } \\
\text { regarder } \\
\text { l'expérience } \\
\text { faite par le } \\
\text { professeur }\end{array}$ & $\begin{array}{l}\text { Lien avec les } \\
\text { observations }\end{array}$ \\
\hline $\begin{array}{l}\text { Réponses aux ques- } \\
\text { tions }\end{array}$ & \multicolumn{3}{|c|}{$\begin{array}{l}\text { écouter et répondre aux questions, copier } \\
\text { les inscriptions du tableau }\end{array}$} & \multicolumn{2}{|c|}{ écouter } & Conclusion \\
\hline $\begin{array}{l}\text { Transition avec la } \\
\text { tâche suivante }\end{array}$ & écouter & écouter & & \multicolumn{2}{|c|}{ écouter } & \\
\hline
\end{tabular}

\section{Des introductions variées}

La durée de l'introduction de la séance est nettement plus longue pour madame Branly que pour mesdames Say et Forest (voir tableau 2). En effet, madame Branly effectue de longs rappels sur l'enseignement de $1^{\text {ère }} \mathrm{S}$ (caractéristique d'une pile, différents types de générateur). Tout est écrit au tableau pour copie, à l'exception du rappel du critère d'évolution qui est évoqué par oral et de façon incomplète dans le groupe 1. Ce rappel fait, au contraire, l'objet d'un écrit au tableau lors d'un bref jeu de questionsréponses avec un élève dans le groupe 2 .

Madame Say situe la séance dans la progression et présente la structure de la séance en deux parties. Un rappel sur le critère d'évolution est effectué avec les élèves lors d'un bref jeu de questions-réponses orales.

Madame Forest engage par de brèves consignes les élèves dans le travail à faire, sans faire mention de la nécessité de choisir une équation chimique figu- 
rant dans le texte pour appliquer le critère d'évolution, ni rappeler celui-ci.

Dès la fin de la première séance madame Branly déplore de ne pas avoir eu le temps de faire tout ce qu'elle avait prévu. Elle justifie en entretien le temps passé sur les rappels d'électricité en déclarant qu'il s'agit de notions fréquemment évaluées au baccalauréat. Ce temps passé incite à dire que l'enseignante a momentanément changé de but, en privilégiant un objectif plus lointain puisque les notions qu'elle fait rappeler ne sont pas utilisées ensuite dans le reste de la séance. L'appel au baccalauréat montre l'influence forte de cet aspect de la composante institutionnelle sur la composante médiative de l'activité de l'enseignante qui fait durer ce moment de dialogue collectif.

Madame Branly et madame Say font toutes les deux un rappel du critère d'évolution. Cette action d'accompagnement classique vise à éviter une perte de temps ultérieure ou une éventuelle absence de réponse en remémorant aux élèves ce qui sera utile par la suite. C'est une préparation cognitive des élèves pour la suite. Nous notons l'ajustement effectué par madame Branly du groupe 1 au groupe 2 : elle passe à un rappel écrit, peut-être en réaction aux flottements éprouvés lors de la mise en œuvre du critère d'évolution dans le groupe 1.

Madame Forest au contraire ne procède à aucun rappel et laisse les élèves face à la difficulté d'avoir à choisir une équation pour débuter la résolution. Il s'agit d'une décision délibérée qu'elle reproduit chaque année comme elle l'a déclaré en entretien. Ce choix montre qu'elle considère que les élèves doivent chercher, se poser des questions sur la nécessité de faire telle ou telle chose pour résoudre, confronter leurs connaissances finalement. Cela participe d'une appropriation de la question posée qui est susceptible de mieux leur faire comprendre la portée de la résolution. Cette façon de procéder donne des indications sur sa conception de l'apprentissage et révèle une imbrication de la composante personnelle de son activité (apprentissage) et de sa composante cognitive (nature de la tâche).

\section{Réalisation des expériences}

Madame Branly et madame Say s'appuient sur la réalisation de l'expérience pour mettre en commun les observations puis répondre aux questions (appliquer le critère d'évolution) tandis que madame Forest met en œuvre le critère d'évolution pour effectuer une prévision du sens d'évolution qu'il s'agit ensuite de confronter aux observations.

Dans la classe de madame Say, ce sont les élèves qui réalisent les expériences en binômes (ce qui allonge notablement la durée), alors que dans celle de madame Branly l'expérience est faite au bureau par une élève qu'elle guide dans le groupe 1 et par elle-même dans le groupe 2. Dans les deux classes, les observations des expériences sont faites et institutionnalisées lors d'une mise en commun, sous forme d'un dialogue collectif, comme les réponses aux questions de la fiche TP.

Alors que madame Branly et madame Forest partagent le fait de ne pas avoir fait faire l'expérience par les élèves, elles n'en partagent pas la raison. Madame Branly procède ainsi pour gagner du temps et faire toutes les expériences prévues dans le temps imparti, tandis que madame Forest a été contrainte par la rupture d'approvisionnement du laboratoire en sulfate de cuivre, qui n'a pas permis de faire autant de solution qu'il aurait été nécessaire pour que les élèves manipulent. Le choix de madame Forest peut être qualifié de contingent et contraint, et illustre l'influence d'un facteur de la composante sociale de son activité sur ses composantes cognitive (changement de tâche) et médiative (changement d'étayage). Pour madame Branly le choix non fortuit résulte d'un compromis entre les composantes institutionnelle (traiter tout ce qui est au programme) et cognitive (voir l'expérience pour répondre aux questions) de son activité.

\section{Absence de questionnement sur la réalisation de la pile}

Pour les trois enseignantes la mise en relation avec la deuxième tâche (la réalisation d'une pile) est rapide, moins d'une minute, voire inexistante (madame Branly, groupe 2), sans que les conditions de cette réalisation ne fassent l'objet d'un questionnement. La nécessité de la séparation des espèces chimiques en couples oxydant-réducteur conjugués pour fabriquer une pile est imposée (ce qui était visible dans les fiches TP) et n'est pas non plus discutée oralement, ce qui aurait pu l'être, notamment lorsque le transfert d'électrons est évoqué en fin de tâche. La transition avec la seconde tâche est faite a minima ou absente. 
Dans la partie suivante nous décrivons de façon plus précise la succession des sous-épisodes dans chaque classe lors de l'interprétation ou de la prévision de la transformation chimique étudiée ${ }^{6}$.

\section{Différences interindividuelles et intra-individuelles}

Nous nous attachons à caractériser les liens établis ou non par les enseignantes entre les observations expérimentales et leur prévision ou leur confirmation à l'aide du critère d'évolution en prenant appui sur le schéma de fonctionnement du modèle thermodynamique comme annoncé antérieurement (cf. paragr. Traitement et analyse des données). Il s'agit de fournir des éléments de réponses aux questions de recherche, pour préciser la place des transferts d'électrons, des modèles, des expériences dans la séance, mais aussi pour repérer des régularités et des éléments de variabilité.

Nous obtenons des schémas de déroulement (voir Fig. 4, 5, 6, 7 et 8) qui présentent la succession chronologique des sous-épisodes dont chacun correspond à la réalisation d'une ou plusieurs sous-tâches inscrites dans un rectangle qui porte un numéro d'ordre chronologique. Les rectangles sont reliés par des flèches. Une flèche en trait plein indique l'ordre de succession des sous-tâches. Une flèche à deux pointes signifie qu'il y a eu une mise en relation explicite lors de la réalisation de deux sous-tâches. La nature de la mise en relation est précisée dans un rectangle avec une bordure double. Une flèche en pointillé indique que la réalisation d'une sous-tâche s'appuie sur le résultat d'une sous-tâche autre que la précédente.

Selon l'approche adoptée par les enseignantes (observations puis utilisation du modèle ou l'inverse) la succession des sous-tâches est différente. Ces schémas révèlent également des variations intraindividuelles d'un groupe à un autre suivant les évènements intervenus, et des variations interindividuelles, en particulier lorsqu'une même approche est mise en œuvre avec des dispositifs expérimentaux différents (cf. tableau 1).

Dans chacun des paragraphes suivants, nous décrivons les principaux sous-épisodes de chaque séance, en détaillant quelques aspects qui permettront de fournir des éléments de réponses aux questions de recherche. Puis nous fournissons une interprétation des raisons de certains choix en lien avec nos questions.

\section{Descriptif des déroulements de madame Branly}

Durant les séances de madame Branly, l'expérience est faite au bureau et le protocole n'est pas totalement respecté puisqu'il n'y a pas de thermomètre mis dans le mélange et que celui-ci n'est pas filtré.

Dans le groupe 1, la réalisation du mélange est faite par une élève au bureau avec des copeaux de cuivre, sous la conduite de l'enseignante. L'absence de poudre de cuivre conduit l'enseignante à sortir de la salle pour en chercher, en vain. Les observations sont difficiles, trop peu de temps s'étant écoulé entre la réalisation de l'expérience et la demande d'observation, ce qui conduit l'enseignante à demander aux élèves de les prévoir (d'où l'appellation « Prev.-Obs » de la sous-tâche 1' de la Fig. 4). Elle montre la lame de zinc à quelques élèves et l'un d'eux suggère " y aura pas un dépôt de cuivre aussi? » sans le décrire. L'écriture de l'équation chimique qui suit est très courte ( 24 secondes), une élève dictant à l'enseignante sans faire référence aux observations (2, Fig. 4). L'enseignante dialogue avec d'autres élèves pour répondre à la deuxième question portant sur le critère d'évolution, qu'elle décompose en sousquestions (3 et 4, Fig. 4). Elle interprète les observations en termes d'oxydation et réduction des espèces chimiques (5 et 6, Fig. 4). Elle justifie la lenteur de la transformation chimique par le fait que le cuivre n'était pas sous forme de poudre ce qui diminue la surface de contact et augmente le temps de réaction ${ }^{7}$. Elle termine en faisant un nouveau rappel sur le critère d'évolution. Alors qu'elle a débuté la lecture de la deuxième partie (la pile Daniell), elle revient sur l'interprétation en termes de transfert d'électrons (7, Fig. 4).

Dans le groupe 2, l'enseignante fait elle-même le mélange avec de la poudre de cuivre ${ }^{8}$. En réponse à la demande d'observation un élève annonce qu'il y a un précipité lorsqu'il voit la poudre de cuivre en suspension dans la solution. L'enseignante sonde alors les élèves sur ce qu'est un précipité avant de faire une expérience pour leur montrer l'apparition d'un précipité (2, Fig. 5) et le définir. Ensuite, comme dans le premier groupe l'enseignante fixe les observations qu'elle attend ( 1 et 2', Fig. 5), intera- 
Figure 4.

Déroulement de la séance du groupe 1 de madame Branly

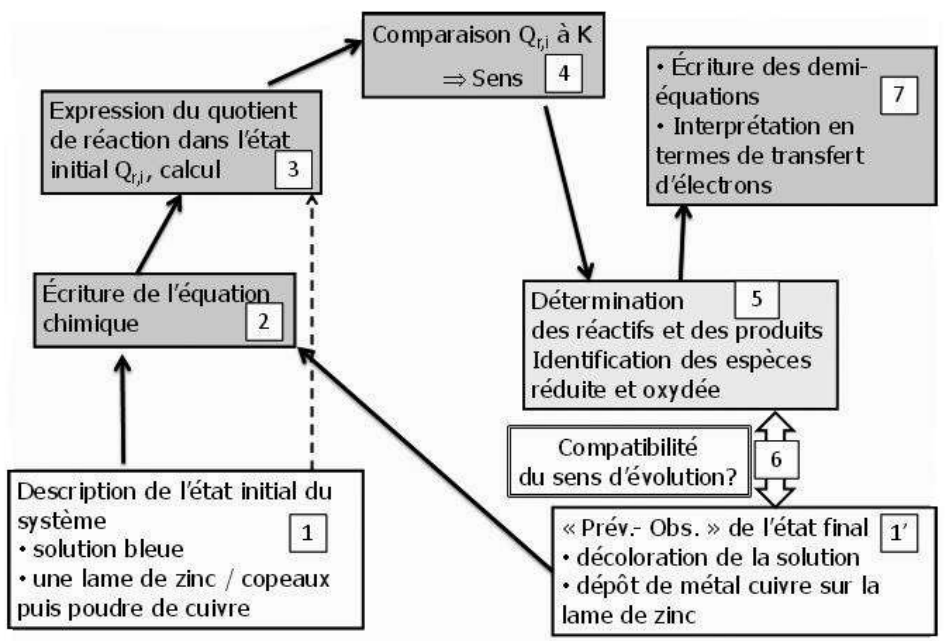

git ensuite rapidement (37 secondes) avec un élève pour l'écriture de l'équation de réaction (3, Fig. 5) dont elle demande la nature pour finir par dire qu'il y a eu un transfert direct d'électrons au sein de la solution (4, Fig. 5). La mise en œuvre du critère d'évolution s'effectue aussi par l'intermédiaire de sous-questions simples ( 5 et 6 , Fig. 5). Elle conclut elle-même sans solliciter les élèves sur le sens d'évolution du système et fait un retour sur l'expérience pour préciser ce qu'il advient des différentes espèces chimiques en attribuant l'aspect noir du dépôt débutant de cuivre sur la lame de zinc à son caractère très divisé ( 7 et 8 , Fig. 5).

Si nous comparons les schémas de déroulement des deux séances de madame Branly (Fig. 4 et 5), trois points sont à noter. Tout d'abord, comme annoncé dans l'analyse a priori, la consommation des ions zinc n'a pas été mise en évidence et les observations n'ont pas été complètes pour le groupe 1 mais ont pu être menées à leur terme pour le groupe 2 . Ensuite, l'interprétation en termes de transfert d'électrons n'est pas située au même moment de la séance : en début de deuxième tâche pour le groupe 1 ( 7 , Fig. 4), dès après l'écriture de l'équation chimique pour le groupe 2 (4, Fig. 5). Enfin, la présence de poudre de cuivre pour le groupe 2 a provoqué un incident didactique au sens de Roditi (2008), une réponse en décalage avec ce que l'enseignante attendait, à la suite duquel madame Branly a clairement dévié de son projet initial et estimé qu'un retour sur le concept de précipité était nécessaire.
Nous interprétons maintenant ces choix et actions en termes de composantes de l'activité de madame Branly. L'absence d'observation concernant les ions zinc ne semble pas être une difficulté pour madame Branly parce que l'observation du léger dépôt de cuivre suffit à constituer l'indice déclencheur du sens d'écriture de l'équation de réaction d'oxydoréduction, exercice auquel les élèves sont rompus nous a-t-elle dit spontanément en entretien. Cela révèle un compromis fait par l'enseignante pour concilier plusieurs impératifs, partir de l'expérience (impératif cognitif dû au type de tâche choisie), ne pas y passer trop de temps (impératif institutionnel) et enrôler les élèves (impératif médiatif).

Nous pensons que le retour tardif (dans le groupe 1) sur la question du transfert d'électrons, point clé de passage à la fabrication d'une pile électrochimique, correspond à un oubli de l'enseignante. Il s'agit d'une conséquence de l'absence de poudre de cuivre, jugée à tort indispensable par l'enseignante. En effet lors de l'entretien, elle a évoqué son état de stress permanent dans le groupe $1^{9}$, car à chaque première mise en œuvre d'un TP, elle doit faire face à des manques de matériel ou dysfonctionnements ${ }^{10}$ qu'elle corrige pour que les expériences puissent être effectuées comme elle l'attend. Elle n'a pas pu venir tout vérifier avant la séance, ayant cours. L'organisation du travail dans l'établissement (emploi du temps de l'enseignante, disponibilité insuffisante de la préparatrice) influe sur l'état d'esprit de l'enseignante et sur le déroulement de la séance de classe. 
Figure 5.

Déroulement de la séance du groupe 2 de madame Branly

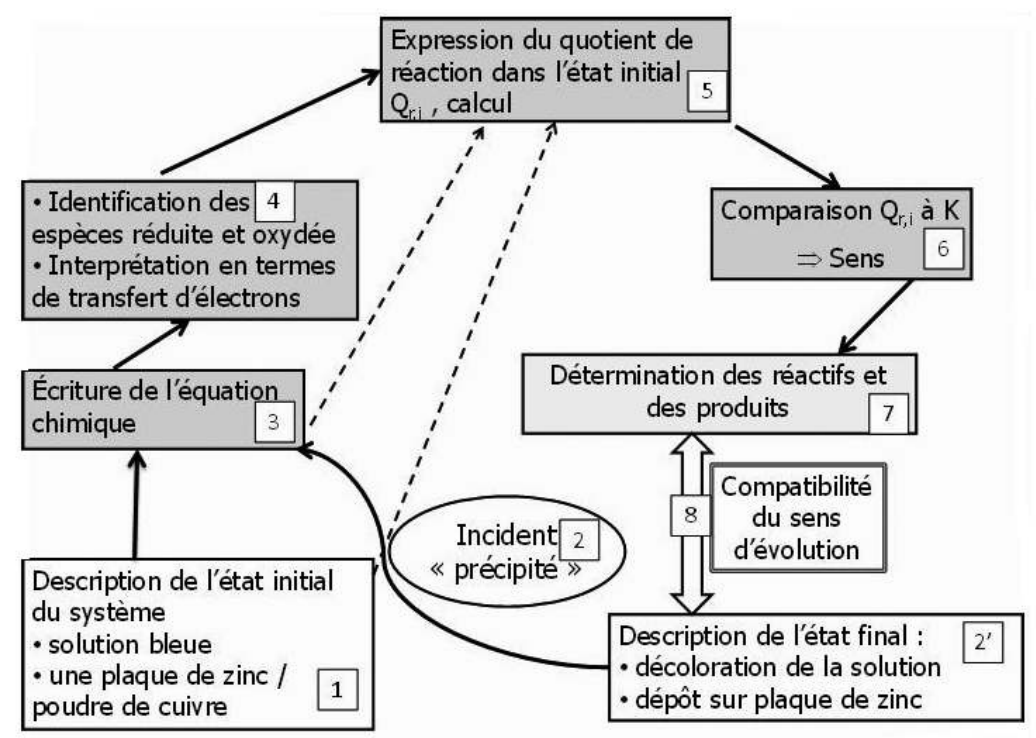

Ce facteur peut être attribué à la composante sociale de l'activité de l'enseignante dont on voit la répercussion sur la composante personnelle et la composante médiative.

L'incident didactique précipité est révélateur de sa vision personnelle des connaissances de chimie que doivent avoir les élèves (une remarque du même type d'une élève dans la classe de madame Say n'a pas été relevée et n'a donné lieu à aucune mise au point).

\section{Déroulement de la séance de madame Say}

Durant la réalisation des expériences par les élèves en binômes, madame Say les observe en silence, répond aux demandes de précision de certains et donne trois indications à toute la classe à trois moments (suite à une question d'élève). Elle indique que le compte-rendu du TP sera à rendre en fin de séance, demande "d'écrire la réaction qui a eu lieu dans le tube deux » et donne la formule des ions zinc. La réponse aux questions s'effectue lors d'un dialogue avec la classe.

Madame Say fait préciser les observations à Carla, (1 et 1', Fig. 6), les fait interpréter en termes d'espèces chimiques consommées et formées (2, Fig. 6) après de longs échanges au cours desquels elle en appelle au principe de conservation des éléments chimiques lorsque les élèves ne se souviennent pas de l'inter- prétation de la deuxième expérience. Puis elle entreprend d'écrire l'équation de réaction au tableau tout en dialoguant avec la classe (3, Fig. 6). Une question sur la nature de la réaction en jeu suscite une réponse immédiate de plusieurs élèves « précipitation ». Elle réagit en déclarant qu'elle ne parle pas de l'expérience avec la soude. Puis une élève parle de transfert d'électrons. Madame Say complète alors au tableau les demi-équations électroniques qu'elle avait écrites auparavant sous l'équation globale (4, Fig. 6), en disant qu'elles ne sont « pas vraiment la réalité » mais qu'il s'agit de « notre modèle de compréhension ». Madame Say engage un nouveau dialogue pour mettre en œuvre le critère d'évolution (5 et 6, Fig. 6), et relie le résultat de cette application du critère avec ce qu'ils observent (7, Fig. 6). En fin de discussion, les élèves ont sollicité (et obtenu) la réalisation de l'expérience inverse, en prenant du cuivre et une solution de sulfate de zinc, pour constater la non évolution du système. Pour assurer la transition avec la réalisation d'une pile, madame Say pose la question «Est-ce que c'est possible de faire réagir deux espèces chimiques qui ne sont pas en contact? » à laquelle les élèves répondent « avec un pont salin » parce qu'ils ont lu la suite du TP, sans expliquer davantage.

Notons tout d'abord la reformulation de la première question de la partie analyse, madame Say indique qu'il faut écrire une équation de réac- 
Figure 6.

Déroulement de la séance de madame Say

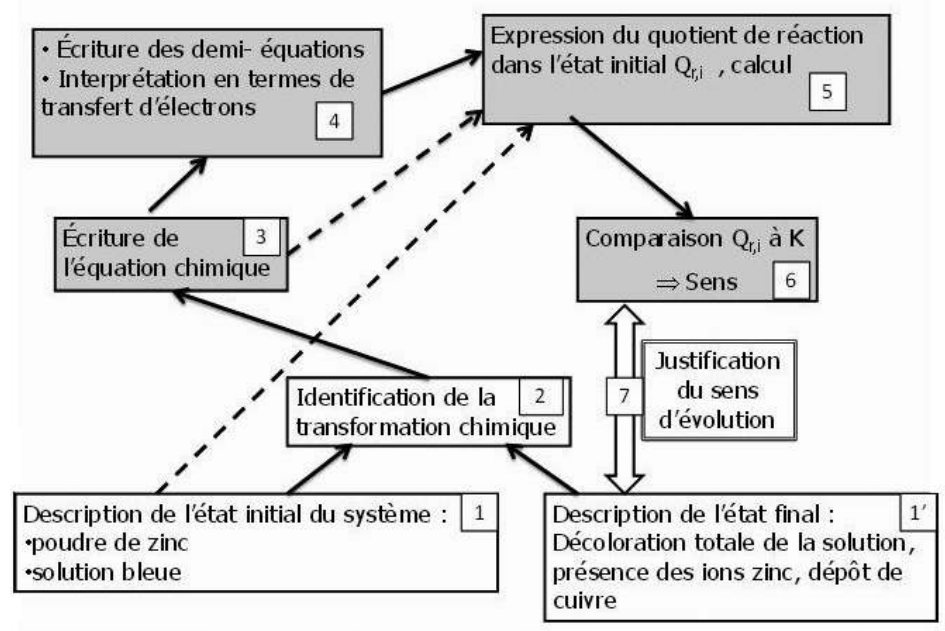

tion pour caractériser la première transformation chimique, nécessaire pour mettre en œuvre le critère, comme nous l'avions dit dans l'analyse a priori. Comme celle-ci le pressentait, les élèves n'ont pas su interpréter la seconde expérience pour identifier un produit de la première. Madame Say a contourné la difficulté en faisant appel au principe de conservation des éléments chimiques, et a donc adapté sa stratégie aux réactions des élèves. L'interrogation sur la nature de la transformation chimique étudiée déclenche une réponse erronée "précipitation » qui montre que les élèves ne semblent pas avoir pleinement conscience que deux transformations chimiques successives ont été réalisées, une par expérience. Dans l'entretien madame Say revient sur cette difficulté récurrente, les élèves «n'arrivent pas à séparer le test de la transformation qu'on étudie » et ne parvient pas à l'interpréter. Cependant, elle justifie son utilisation : « c'est aussi pour réinvestir ce qu'ils ont fait puisqu'on leur a appris au collège à faire des tas de tests il faut qu'à un moment ils comprennent que ça sert à quelque chose » sans pour autant détailler le fonctionnement du test (voir Fig. 2) en classe, qui n'a pas tout à fait le statut d'une transformation chimique.

En entretien madame Say a spontanément déclaré qu'elle n'avait pas prévu de faire "l'expérience inverse » sans dire pourquoi elle avait cédé à la pression des élèves. Cependant ce choix est cohérent avec notre analyse de la fiche TP, de même que son commentaire aux élèves sur le rôle d'un élément de modèle (les demi-équations électroniques). Ils constituent une autre indication de l'image de la chimie qu'elle souhaite donner, la chimie comme science expérimentale, utilisatrice de modèles interprétatifs et prédictifs, puisque « l'expérience inverse » ne doit conduire à aucun changement. Ceci est un autre exemple de l'influence de la composante personnelle de l'activité de l'enseignante sur sa composante cognitive.

\section{Déroulement des séances de madame Forest}

Les deux séances de madame Forest commencent par une phase de recherche individuelle des élèves qui doivent prévoir l'évolution du système correspondant au mélange décrit sur leur fiche TP.

Dans le groupe 1, madame Forest prépare le mélange sur une annexe du bureau pendant que les élèves cherchent. Après avoir circulé en silence et fait des commentaires personnalisés à certains binômes (sur la nécessité de choisir une équation de réaction et de prendre en compte les états physiques des espèces pour l'écriture du quotient de réaction), elle envoie un élève au tableau, Léo, pour corriger. Celui-ci demande alors « je fais tout? " pour indiquer qu'il a fait l'exercice avec les deux équations. Madame Forest lui répond d'en prendre une seule. Léo, en silence, écrit l'équation de réaction qu'il a choisie (1, Fig. 7 ), le quotient de réaction associé 
et son calcul (2' et 2, Fig. 7), le sens d'évolution prévu (3, Fig. 7), l'espèce oxydée et l'espèce réduite (4, Fig. 7) pour lesquelles il se trompe. Madame Forest engage alors un dialogue avec lui pour qu'il se corrige et écrive les demi-équations électroniques, elle revient ensuite sur le calcul erroné des concentrations dans le quotient de réaction. Léo reparti à sa place, elle débute un dialogue avec la classe pour prévoir les observations (5, Fig. 7), et invoque la cinétique de la transformation pour justifier la difficulté de noter le moindre changement. Elle discute des améliorations à apporter pour modifier la cinétique avant de conclure sur une explicitation de ce qu'est un transfert direct d'électrons en commentant ce qui figure sur leur fiche TP (6, Fig. 7).

Dans le groupe 2, elle n'envoie pas d'élève corriger au tableau. Mais elle entreprend de guider le raisonnement par des indications fournies à toute la classe, d'abord en leur demandant de faire attention aux états physiques, puis de choisir une équation (1, Fig. 8), et enfin d'établir l'expression du quotient de réaction initial sans tenir compte des solides (2, Fig. 8). Ces indications surviennent après qu'elle a rencontré une difficulté spécifique en regardant les écrits de plusieurs binômes. La difficulté suivante concerne le calcul des concentrations qu'elle constate en regardant le travail d'Antoine. Elle engage avec lui un dialogue à voix suffisamment haute pour être entendue de tous, en détaillant oralement le calcul (2' et 2, Fig. 8). Elle poursuit le raisonnement en sollicitant une réponse d'élève de temps à autre pour prévoir le sens d'évolution (3, Fig. 8), et pour déterminer l'espèce chimique réduite, l'espèce oxydée (4, Fig. 8). Elle écrit au tableau les prévisions d'observation, qu'elle leur fait constater en montrant le bécher comportant le mélange réalisé dans le groupe 1 ( 5 et 6 , Fig. 8). Elle conclut sur le caractère direct du transfert d'électrons (7, Fig. 8).

La comparaison des schémas de déroulement des deux séances de madame Forest (Fig. 7 et 8) fait apparaître une forte similitude de structure même si deux différences sont à noter. La première concerne l'état final : dans le groupe 1 (Fig. 7), il s'agit uniquement d'une prévision à l'aide du modèle puisque la validation expérimentale n'a pu être menée à son terme, alors que pour le groupe 2 , cette dernière a bien eu lieu. La deuxième a trait aux demi-équations électroniques qui ne sont pas évoquées dans le groupe 2 (Fig. 8). De plus, si l'on compare cette fois la mise en ouvre de ces déroulements à la structure quasi identique, on constate une forte différence, il n'y a pas eu d'écriture au tableau du raisonnement mettant en ouvre le critère d'évolution, ni des demiéquations électroniques dans le groupe 2 .

En entretien nous l'avons interrogée sur cette variation dans sa stratégie : elle a présenté l'envoi d'un élève au tableau comme n'étant pas planifié et résultant de l'humeur du moment. Cette déclaration montre que madame Forest ne considère pas devoir conduire les deux séances consécutives exactement de la même façon et qu'elle s'autorise des variations autour d'un canevas commun, dont on peut penser

Figure 7.

Déroulement de la séance de madame Forest avec le groupe 1

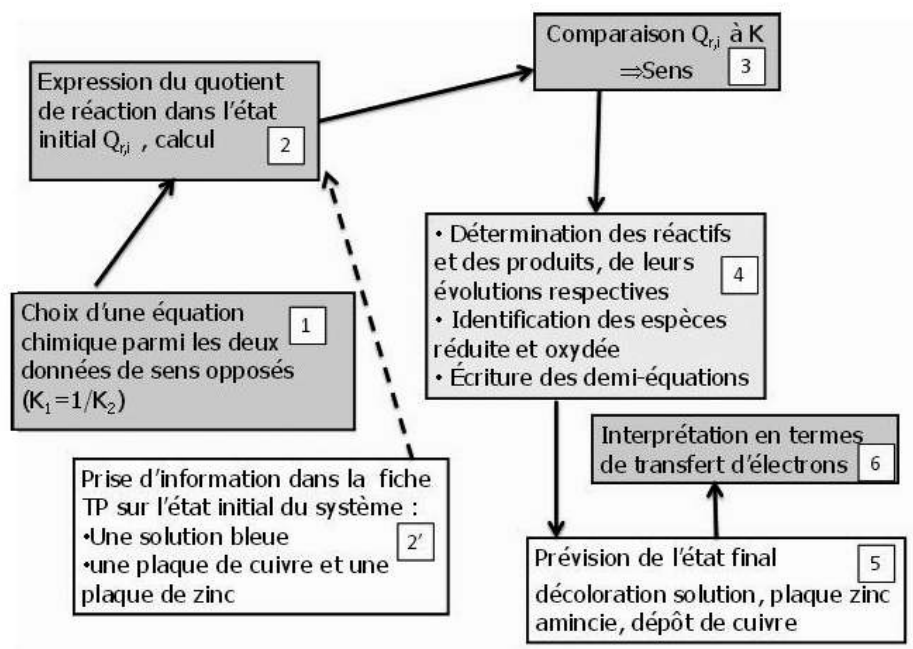


Figure 8.

Déroulement de la séance de madame Forest avec le groupe 2

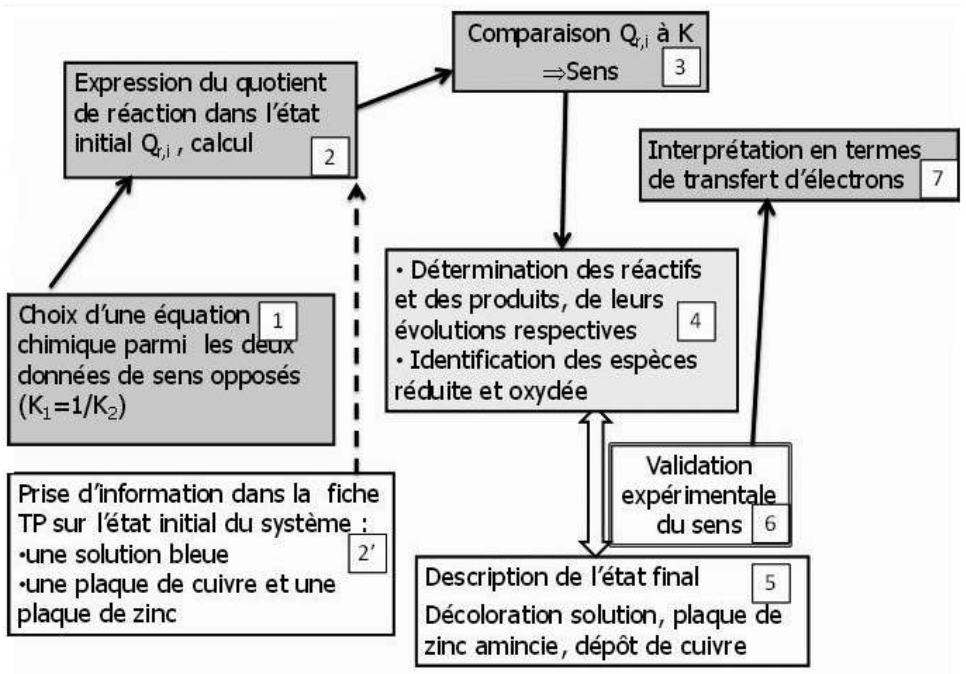

qu'elles résultent d'une interaction entre la composante personnelle et la composante médiative de son activité. Cependant les consignes qu'elle a fournies dans le groupe 2 pour guider le raisonnement des élèves sont plus directives, elle dit explicitement de choisir une équation, pour éviter les interrogations soulevées par ce point dans le groupe 1. Ces modifications du discours peuvent être interprétées comme une adaptation différée de ses actions aux réactions des élèves.

\section{Comparaison des déroulements}

Ces schémas (Fig. 4 à 8 ) nous permettent de visualiser le moment où le transfert d'électrons est mentionné. Pour madame Say et madame Branly (groupe 2), la mention du transfert survient après l'écriture de l'équation de réaction et constitue alors une interprétation logique de celle-ci. Mais elle n'est pas reprise en fin d'épisode pour faire la transition avec la réalisation de la pile à suivre. Madame Forest et madame Branly (groupe 1) mentionnent le transfert en fin d'épisode, ce qui pourrait être plus propice pour faire un lien avec la tâche suivante, mais ce n'est pas non plus le cas. Le transfert d'électrons est dit être direct, puis indirect dans le dispositif de la pile qui est simplement décrit. Pour les trois enseignantes, la nécessité de la mention du transfert d'électrons pour répondre à une interrogation qui n'a pas été formu- lée (comment obtenir un courant électrique à partir d'une transformation chimique) n'apparait pas.

Ces schémas montrent également, la stabilité de la succession des sous-tâches pour madame Forest d'un groupe à l'autre tandis qu'ils révèlent des variations pour madame Branly.

Madame Branly et madame Say ont adopté la même approche, observations puis utilisation du modèle. Seule madame Say a procédé à une identification de la transformation chimique avant de faire écrire l'équation de réaction, et n'a pas fait appel à un savoir-faire antérieurement travaillé (comme madame Branly) mais à une réflexion basée sur un principe fondamental de la chimie et faisant appel aux habitudes de la classe.

\section{SYNTHÈSE ET DISCUSSION}

Nous reprenons les résultats de nos analyses en les regroupant pour répondre à nos questions de recherche. Notre étude de l'activité de ces trois enseignantes, limitée à la première partie des séances observées, s'attache dans un premier temps à caractériser la mise en œuvre du contenu choisi par les enseignantes pour susciter l'activité des élèves.

À travers notre première question de recherche nous interrogeons la façon dont les enseignantes abordent le passage d'une transformation chimique spontanée à une pile électrochimique. L'examen des 
Isabelle Kermen \& Philippe Colin

fiches TP et celui des déroulements montrent que les trois enseignantes font le même choix de ne pas questionner la juxtaposition d'une transformation chimique spontanée et de la pile Daniell, et d'imposer le dispositif expérimental de la pile (séparation des constituants). Le rôle du transfert des électrons est évoqué à des moments différents selon les enseignantes mais il n'est pas un élément de discussion, il est simplement qualifié de « direct» ou « indirect». Il pourrait s'agir de manifestation d'habitudes professionnelles en accord avec ce que l'on trouve dans le document d'accompagnement du programme et dans les manuels scolaires.

Notre deuxième question de recherche vise à déterminer comment le dispositif expérimental choisi et le questionnement associé orientent le déroulement de la première partie des séances et l'activité des élèves. Le dispositif expérimental utilisé par mesdames Branly et Forest (quatre espèces chimiques initialement) présente l'inconvénient de ne pas permettre la mise en évidence de la formation d'ions zinc, la décoloration de la solution étant peu visible, tout comme la formation de cuivre. Ces difficultés, amplifiées dans le cas de madame Branly par le fait qu'elle ne respecte pas le protocole prévu (pour gagner du temps), contribuent à rendre les observations secondaires. Madame Forest traite la difficulté en initiant un court dialogue sur les améliorations à apporter pour augmenter la cinétique tandis que madame Branly induit la réponse attendue, qui lui sert de déclencheur pour débuter l'écriture de l'équation de réaction.

Au contraire, madame Say, dont le dispositif expérimental ne met en jeu que deux espèces chimiques ${ }^{11}$, a prévu de caractériser les deux espèces qui se forment. Mais les indices expérimentaux n'étant ni suffisants (problème de l'aspect de la poudre dans le tube), ni accompagnés des connaissances associées (identification des ions zinc), les seules observations ne lui permettent pas de faire l'inventaire des espèces présentes dans l'état final. Elle choisit alors de « réfléchir » avec les élèves. Elle recourt à la conservation des éléments chimiques pour conforter et légitimer les observations.

Malgré les difficultés des observations et la composition initiale du système, rien ne s'opposait à ce que madame Branly fasse un état des lieux détaillé du système final comme madame Say, quitte à s'appuyer sur la conservation des éléments chimiques comme elle, pour pallier l'absence de caractérisation des ions zinc. Manifestement c'est une option que madame Branly n'a pas envisagée, préférant s'appuyer sur la donnée des couples oxydant-réducteur et réactiver un savoir-faire travaillé en début d'année, l'écriture d'une équation d'oxydoréduction, savoir-faire qu'elle estime utile d'entretenir dans l'optique de l'examen.

Le questionnement, qui accompagne les dispositifs expérimentaux différents, suscite des activités différentes des élèves. Ils doivent réfléchir seuls et produire une prévision chez madame Forest, qui, passant de table en table, leur fournit des aides ponctuelles. Madame Say procède de la même manière pour les tâches expérimentales qu'elle a prévues. Ces deux enseignantes laissent des moments de silence où il est patent que les élèves travaillent seuls. Plusieurs élèves dans ces deux classes posent des questions aux enseignantes, demandent des précisions, autant de signes d'une appropriation de la tâche et donc d'une activité effective. Dans la classe de madame Branly, les traces d'une activité cognitive des élèves sont plus rares : ils n'ont pas à réaliser de tâche en autonomie et l'enseignante pose plusieurs questions dont la formulation laisse peu d'incertitude sur la réponse attendue (effet Topaze).

Le questionnement de nature théorique qui accompagne ces dispositifs n'a pas le même rôle dans les trois séances. Dans la classe de madame Forest, il constitue l'entrée des élèves dans la séance (non sans difficulté si l'on se fie aux consignes qu'elle leur fournit en cours de réflexion) alors qu'il succède à une phase expérimentale pour les élèves de ses collègues. Madame Forest défend l'idée de mettre les élèves face à la difficulté du choix de l'équation de réaction au début de la résolution à la fois pour des raisons cognitives (conception de l'apprentissage) et épistémologiques (mettre en exergue le rôle du modèle).

Notre troisième question de recherche concerne le rôle attribué au niveau expérimental et à celui des modèles dans ces séances. Nous avons relevé deux approches : l'une s'appuyant sur le modèle pour prévoir une transformation chimique (madame Forest), l'autre partant des observations expérimentales pour justifier la transformation chimique $a$ posteriori avec le modèle. Madame Branly et madame Forest utilisent à peu de chose près (poudre ou lame de cuivre) le même dispositif expérimental à quatre espèces chimiques initiales. La question de la détermination du sens d'évolution n'est donc pas artificielle. Leurs stratégies sont aux antipodes l'une de 
l'autre : appui sur l'expérience ou appui sur le modèle pour décider de ce sens. Le rôle attribué au modèle est alors, soit justificateur de ce que l'expérience montre, soit au contraire prédicteur de l'expérimental. Ce choix peut relever d'influences diverses : la conception du rôle des modèles (madame Forest, voir plus haut) ou la conformité à ce qui figure dans le document d'accompagnement du programme. Le second cas, qui constitue une forme de respect des recommandations institutionnelles, concerne madame Branly, qui est la seule dans la dernière partie de sa fiche à avoir repris in extenso une autre expérience figurant dans ce document (la pile usée).

Madame Say, accorde une place plus grande que ses collègues à l'aspect expérimental, mais ne minimise pas pour autant le rôle du modèle. Pourtant dans le cadre d'un contrat didactique standard, il ne fait aucun doute qu'une fois l'équation de réaction écrite, il ne pouvait que se former ce qui a été observé (les espèces écrites à droite dans l'équation de réaction). La mise en œuvre du critère d'évolution est une vérification qui n'a pas de caractère de nécessité. Néanmoins, la conduite à terme du raisonnement montre son souci de rigueur.

Nous abordons maintenant notre quatrième interrogation concernant la recherche de régularités et de variabilités parmi ces trois enseignantes. Nous avons noté quelques caractéristiques communes aux trois enseignantes. Elles ont choisi d'étudier une première transformation chimique interprétée par l'équation de réaction de la pile Daniell, objet de la deuxième tâche, et ne questionnent pas le passage du premier dispositif expérimental à celui d'une pile. Bien que les élèves soient en salle de TP, où ils pourraient être en autonomie et devoir produire un document ou une réponse argumentée et élaborée à une question en fin de séance, les moments de dialogue collectif précèdent l'institutionnalisation par l'enseignante. On assiste à une forme de cours dialogué, de pédagogie interrogative (Houssaye, 2014), où l'enseignante est la source du savoir et conduit les échanges de façon plus ou moins dirigiste, les élèves étant rarement générateurs de questions. L'enseignante fait preuve d'une tutelle plus ou moins stricte (DumasCarré \& Weil-Barais, 1998), la forme extrême, si l'on peut dire, réduisant les élèves à ne prononcer qu'un mot voire une syllabe (cas de madame Branly étudié par ailleurs [Kermen $\&$ Barroso, 2013]), et produisant un discours à trous déjà repéré par Venturini et ses coauteurs (2007). Ces caractéristiques communes constituent-elles des régularités? Il est difficile de l'affirmer dans la mesure où l'étude ne porte que sur trois enseignantes et un nombre réduit de séances de classe, mais elles en sont des prémisses.

Nos analyses révèlent également des éléments de variabilités entre enseignantes, et pour une même enseignante entre deux séances ou entre son projet de séance et la réalisation. Tout d'abord malgré le contexte très contraint d'un programme de classe d'examen, nous avons fait état de la diversité des approches conduites par les enseignantes concernant la place du modèle, des observations et l'implication réflexive des élèves. Cela illustre les marges de manœuvre que les enseignantes investissent. Ces variabilités sont également les adaptations réalisées par les enseignantes par rapport à leur projet de séance (pas de manipulation élève pour madame Forest), un changement de mode de travail entre deux séances (élève au tableau ou pas, correction écrite au tableau ou pas pour madame Forest), les ajustements effectués pour tenir compte des réactions des élèves. Ces ajustements vont des consignes ou remarques données au groupe classe (après prise d'information de l'enseignante auprès des binômes) lors des phases de travail individuel à des improvisations (incident "précipité », réalisation de « l'expérience inverse ») ou à des changements de discours suivant la réalisation ou non des observations.

Pour terminer la synthèse des analyses effectuées, nous regroupons celles qui examinent les raisons que les enseignantes ont données à certains de leurs choix ou que nous avons inférées. Pour comprendre ces raisons nous avons cherché à déterminer quels facteurs attachés aux diverses composantes de l'activité de l'enseignante (quelles contraintes mais pas uniquement) modulent ces choix. Il s'agit de révéler les logiques d'action des enseignants, qui contribuent à l'atteinte des objectifs qu'elles se sont fixés au sein du réseau de contraintes locales dans lequel elles agissent.

Nous l'avons montré à plusieurs reprises, certains choix s'expliquent en invoquant la part prépondérante d'une composante de l'activité de l'enseignante ou au contraire l'imbrication de plusieurs composantes. Le programme, dans une classe d'examen, constitue un déterminant institutionnel des pratiques central, ce qui ne constitue pas une surprise. Aucune de ces enseignantes n'y "échappe ", mais la façon dont chacune conçoit et met en œuvre la première tâche est révélatrice aussi de l'influence de la compo- 
sante personnelle sur les composantes cognitive et médiative, puisqu'au final ce sont des séances très différentes auxquelles nous assistons. Nous pouvons esquisser des logiques d'action propre à chacune des enseignantes. Madame Say pour qui la réalisation d'expériences par les élèves est incontournable semble soucieuse de donner une image expérimentale de la chimie, science utilisatrice de modèles. Madame Branly est orientée vers l'accomplissement de la tâche, faire l'expérience et l'exploiter pour répondre rapidement aux questions de la fiche, pour parvenir à traiter tout ce qui a été prévu, l'objectif ultime étant la réussite à l'examen (qu'elle invoque à plusieurs reprises dans la séance et dans les entretiens $^{12}$ ). Madame Forest accorde beaucoup d'importance à la réflexion théorique, et amène les élèves à questionner le rôle d'un modèle (choix de l'équation), ce qui la conduit à s'accommoder sans trop de difficulté d'un aléa conjoncturel, la rupture d'approvisionnement du lycée en sulfate de cuivre.

Cette rupture d'approvisionnement peut être rattachée à l'organisation du travail dans le lycée et plus précisément ici aux relations de travail des enseignantes avec les partenaires que sont les préparateurs de chimie. La gestion de l'approvisionnement en produits chimiques qui leur incombe a un effet sur les séances de madame Forest et madame Branly. Nous considérons qu'il s'agit là d'un facteur relevant de la composante sociale de l'activité de chaque enseignante qui rejaillit sur leur activité. Une des originalités de cette étude par rapport à d'autres études des pratiques d'enseignants (de mathématiques) utilisant le cadre de la double approche didactique et ergonomique réside dans la mobilisation d'un facteur associé à la composante sociale de l'activité des enseignantes autre que le milieu social défavorisé des élèves (Butlen et al., 2002; Chesnais, 2011), mais il apparait aussi que ce facteur n'a qu'une influence très locale, puisqu'un défaut de produits chimiques ne survient pas à chaque séance.

Cette étude présente certaines limites, sa brièveté dans le temps, le faible nombre d'enseignantes observées, son contenu disciplinaire restreint eu égard à la brièveté temporelle, la conduite des entretiens (aspect méthodologique évoqué précédemment), mais elle permet cependant de décrire des enseignants de physique-chimie au travail, de les comprendre et de proposer des pistes d'explication (Talbot \& Arrieu-Mutel, 2012). Elle montre aussi que l'utilisation d'un cadre théorique élaboré en didactique des mathématiques permet, moyennant quelques adaptations, l'étude de l'activité d'enseignants d'une autre discipline.

\section{PERSPECTIVES}

Cette étude de cas est révélatrice des stratégies à l'œuvre en classe d'examen à une époque où le programme était très détaillé avec des horaires types et un document d'accompagnement proposant des ressources applicables sans modification (« clés en main »). Elle constitue une forme de témoignage des usages en vigueur dans ces conditions. Malgré la contrainte forte du baccalauréat, les séances présentent des différences notables à la fois en raison des choix cognitifs et pédagogiques des enseignantes mais aussi en raison de leur façon personnelle de les mettre en ouvre. Le programme en vigueur depuis l'automne 2012 en terminale, est beaucoup moins précis et détaillé quant à l'articulation des contenus que le précédent et fait une large place à la conduite d'une démarche scientifique par les élèves. Il serait intéressant de voir si les enseignants ont adapté leurs stratégies et selon quelles modalités.

Une autre perspective pourrait porter sur l'utilisation des résultats obtenus et des outils méthodologiques employés dans le cadre d'une formation à l'analyse des pratiques. Même si les programmes ont changé, le caractère typé de chacune des trois enseignantes constitue un terrain propice à la réflexion des enseignants sur des points d'ordres didactique et pédagogique. 


\section{NOTES}

1. Certaines sont valables aussi pour la physique. Les enseignants en France enseignent les deux disciplines.

2. Seules les traces de cette activité sont accessibles à l'observateur-chercheur.

3. Pour un nombre d'élèves inférieur à 24 , la classe n'est pas dédoublée en TP.

4. Le transfert des électrons vers les espèces chimiques ou inversement s'effectue à l'interface métal-solution.

5. En classe de terminale, ce qui figure dans la colonne « compétences exigibles » du programme constituait les connaissances et raisonnements évalués au baccalauréat. Il n'est pas envisageable qu'un enseignant n'aborde pas ces points.

6. Dans les tableaux 3 et 4 , cela correspond aux phases institutionnalisation des observations et réponses aux questions d'une part, prévision du sens d'évolution, lien avec les observations et conclusion d'autre part.

7. Cependant cette explication n'a pas lieu d'être dans la mesure où le cuivre n'est pas un réactif mais un produit de la transformation et qu'on obtiendrait le même résultat sans cuivre.

8. La poudre a été apportée par la préparatrice au cours de la séance du groupe 1 .

9. Stress qui disparait dans le groupe 2 puisqu'elle a réglé tous les problèmes matériels, nous a-t-elle dit.

10. D'autres problèmes de cet ordre surviennent dans la suite de la séance.

11. Potentiellement réactives.

12. Elle est la seule des trois à le faire avec autant d'insistance.

\section{RÉFÉRENCES}

Barde, N., Barde, M., Besnard, V., Caillet, D., Couture, E., \& Jackel, L. (2002), Chimie Term S. Paris : Hachette.

Bruner, J. S. (1996). Le rôle de l'interaction de tutelle dans la résolution de problème. Dans J. S. Bruner, Le développement de l'enfant : savoir faire, savoir dire ( $5^{\mathrm{e}}$ éd., p. 261-280). Paris, France: Presses universitaires de France.

Butlen, D., Peltier-Barbier, M.-L., \& Pézard, M. (2002). Nommés en REP, comment font-ils ? Pratiques de professeurs d'école enseignant les mathématiques en REF. Contradictions et cohérence. Revue française de pédagogie, 140(1), 41-52.

Chesnais, A. (2011). Apprentissages en mathématiques en sixième : contextes différents, pratiques différentes et inégalités. Revue française de pédagogie, (176), 57-72.

Clot, Y. (1999). La fonction psychologique du travail. Paris : Presses universitaires de France.
Cross, D. (2010). Action conjointe et connaissances professionnelles de l'enseignant. Éducation et Didactique, 4(3), 39-60.

Dumas-Carré, A., \& Weil-Barais, A. (1998). Tutelle et Médiation dans l'enseignement scientifique. Berne : Peter Lang.

Goigoux, R. (2007). Un modèle d'analyse de l'activité des enseignants. Éducation et Didactique, 1(3), 47-69.

Houssaye, J. (2014). La pédagogie traditionnelle. Paris, France : éditions Fabert.

Kermen, I., \& Barroso, M. T. (2013). Activité ordinaire d'une enseignante de chimie en classe de terminale. Recherches en Didactique des Sciences et des Technologies, (8), 91-114.

Kermen, I., \& Colin, P. (2014). Comparaison de l'activité de trois enseignantes lors d'une séance sur les piles en classe de terminale. Communication présentée aux 8 e rencontres scientifiques de l'Ardist, Marseille, 18(2), p. 115-123.

Kermen, I., \& Méheut, M. (2008). Mise en place d'un nouveau programme à propos de l'évolution des systèmes chimiques : impact sur les connaissances professionnelles d'enseignants. Didaskalia, (32), 77-116.

Kermen, I., \& Méheut, M. (2011). Grade 12 French Students' use of a Thermodynamic Model for Predicting the Direction of Incomplete Chemical Changes. International Journal of Science Education, 33(13), 1745-1773.

Leplat, J., \& Hoc, J.-M. (1983). Tâche et activité dans l'analyse psychologique des situations. Cahiers de psychologie cognitive, 3(1), 49-63.

Masselot, P., \& Robert, A. (2007). Le rôle des organisateurs dans nos analyses didactiques de pratiques de professeurs enseignant les mathématiques. Recherche et formation, (56), 15-32.

Ministère de l'éducation nationale (1999). Bulletin Officiel hors-série $n^{\circ} 6$. Paris.

Ministère de l'éducation nationale (2001). Bulletin Officiel spécial $n^{\circ} 4$. Paris.

Ministère de l'Éducation nationale, de la Jeunesse et de la Vie associative (2011). Bulletin Officiel spécial $n^{\circ} 8$. Paris.

Pastré, P. (2007). Quelques réflexions sur l'organisation de l'activité enseignante. Recherche et formation, (56), 81-93.

Robert, A. (2004). Que cherchons-nous à comprendre dans les pratiques des enseignants? Dans M.-L. PeltierBarbier, Dur d'enseigner en ZEP : analyse des pratiques de professeurs des écoles enseignant les mathématiques en Réseaux d'éducation prioritaire (p. 15-32). Grenoble : La Pensée Sauvage.

Robert, A. (2006). Une méthodologie pour décrire des déroulements de séances de classe à partir de vidéo dans des recherches sur les pratiques d'enseignants de mathématiques au collège et au lycée. Dans M.-J. Perrin-Glorian \& Y. Reuter (éd.), Les méthodes de recherche en didactique (p. 191-202). Villeneuve d'Ascq : Presses universitaires du Septentrion. 
Robert, A. (2008a). La double approche didactique et ergonomique pour l'analyse des pratiques d'enseignants de mathématiques. Dans F. Vandebrouck (éd.), La classe de mathématiques: activités des élèves et pratiques des enseignants (p. 59-68). Toulouse : Octarès Editions.

Robert, A. (2008b). Sur les apprentissages des élèves : une problématique inscrite dans les théories de l'activité et du développement. Dans F. Vandebrouck (éd.), La classe de mathématiques: activités des élèves et pratiques des enseignants (p. 33-44). Toulouse : Octarès Editions.

Robert, A. (2012). Une analyse qualitative du travail des enseignants de mathématiques du second degré en classe et pour la classe : éléments méthodologiques. Communication au colloque "Les méthodes d'analyse des pratiques d'enseignement : un regard comparatif". XVII $^{\mathrm{e}}$ congrès mondial AMSE, recherche en éducation et en formation : enjeux et défis aujourd'hui, 3-8 juin 2012, Reims : université de Reims.

Robert, A., \& Rogalski, J. (2002). Le système complexe et cohérent des pratiques des enseignants de mathématiques: Une double approche. Canadian Journal of Science, Mathematics and Technology Education, 2(4), 505-528.

Robert, A., \& Vivier, L. (2013). Analyser des vidéos sur les pratiques des enseignants du second degré en mathématiques : des utilisations contrastées en recherche en didactique et en formation de formateurs - quelle transposition? Éducation et Didactique, 7(2), 115-144.
Roditi, É. (2008). Des pratiques enseignantes à la fois contraintes et personnelles et pourtant cohérentes. Dans F. Vandebrouck (éd.), La classe de mathématiques: activités des élèves et pratiques des enseignants (p. 73-93). Toulouse : Octarès Editions.

Rogalski, J. (2003). Y a-t-il un pilote dans la classe ? Une analyse de l'activité de l'enseignant comme gestion d'un environnement dynamique ouvert. Recherches en didactique des mathématiques, 23(3), 343-388.

Rogalski, J. (2012). Théorie de l'activité et didactique. Pour l'analyse conjointe des activités de l'enseignant et de l'élève. Jornal Internacional de Estudos em Educação Matemática, 5(1). Consulté à l'adresse [http://pgsskroton.com.br/seer/index.php/jieem/article/view/112].

Talbot, L., \& Arrieu-Mutel, A. (2012). Décrire, comprendre et expliquer les pratiques d'enseignement d'un professeur de lycée. Éducation et Didactique, 6(3), 65-95.

Tiberghien, A. (2012). Analyse d'une séance de physique en seconde : quelle continuité dans les pratiques? Éducation et Didactique, 6(3), 97-123.

Tomasino, A. (dir.) (2002). Chimie terminale S. Paris : Nathan.

Vandebrouck, F. (éd.) (2013). Mathematics Classrooms. Rotterdam, The Netherlands: SensePublishers.

Venturini, P., Calmettes, B., Amade-Escot, C., \& Terrisse, A. (2007). Analyse didactique des pratiques d'enseignement de la physique d'une professeure expérimentée. Aster, (45), 211-234. 